\title{
Global existence of a weak solution to 3d stochastic Navier-Stokes equations in an exterior domain
}

\author{
Takeshi Taniguchi
}

\begin{abstract}
In this paper we consider the existence of a weak solution to a 3d stochastic Navier-Stokes equation perturbed by a noise $g(X(t)) d W$, where $W(t)$ is a cylindrical Wiener process, in an exterior domain $D$ :$$
d X(t)=[-A X(t)+B(X(t))] d t+g(X(t)) d W(t),
$$

where $A=-P_{2} \Delta$ is the Stokes operator and $g$ satisfies some conditions.

Mathematics Subject Classification (1991). Primary 76D05; Secondary $76 \mathrm{M} 30,60 \mathrm{H} 15$.
\end{abstract}

\section{Introduction}

In this paper we consider the existence of a weak solution to a $3 d$ stochastic Navier-Stokes equation in an exterior domain $D$ with smooth boundary $\Gamma$ :

$$
\left\{\begin{array}{l}
d X(t)=[\Delta X(t)+(X(t), \nabla) X(t)-\nabla p] d t+g(X(t)) d W(t), \\
\operatorname{div} X=0 \text { in }[0, \infty) \times D, \\
X(t, x)=0 \text { on }[0, \infty) \times \Gamma, X(0)=\phi,
\end{array}\right.
$$

where $X$ is the velocity field of the fluid, $p$ the pressure, $\phi$ the initial velocity field, $g(X(t))$ the intensity of the external noise.

In the last years the existence of the mild solutions and weak solutions, and martingale solutions to the two dimensional or three dimensional stochastic Navier-Stokes equations have been extensively investigated. We emphasize that the stochastic Navier-Stokes equations on a bounded domain or $R^{d}$ $(d=2,3)$ have been well discussed by many authors(Capinski and Gatarek [1], Da Prato and Zabczyk [8], Flandoli and Gatarek [10], Flandoli [11], Mikulevicius and Rozovskii [19] and references therein). But it is seemed that there

This paper is in final form and no version of it will be submitted for publication elsewhere. 
exist a few papers about $2 \mathrm{~d}$ stochastic Navier-Stokes equations in unbounded domain (see e.g. Sritharan and Sunder [24], Brzezniak and Li [1], Taniguchi [27] and references therein). Very recently global existence of a strong solution to a 3-dimensional stochastic Navier-Stokes equation with an additive noise in an exterior domain was considered in [26]. See also Brzezniak and Motyl [2], Brzezniak and Motyl [3], Capinski and Peszat [6].

On the other hand one can find many papers about the existence of weak solutions to the deterministic Navier-Stokes equations. For the case on a bounded domain see e.g. Fujita and Kato [13], Temam [29], Sohr [25], and references therein. For the case on an exterior domain see e.g. Miyakawa [20], Miyakawa and Sohr [14] and therein references. For the case on $R^{d}$ see e.g. Kato [17].

In this paper we investigate the existence of weak solutions to the $3 \mathrm{~d}$ stochastic Navier-Stokes equation perturbed by a additive noise and a multiplicative noise in an exterior domain $D$ following Miyakawa and Sohr [14].

The Miyakawa and Sohr Theorem (Miyakawa and Sohr[14]). Let a $\in$ $L_{\sigma}^{2}(D)$ and $f \in L^{2}\left(0, T ; L_{\sigma}^{2}(D)\right)$ for all $T>0$. Then there exists a weak solution $u$ to a Navier-Stokes equation:

$$
\begin{aligned}
u_{t}-\Delta u+(u, \nabla) u+\nabla p & =f \quad \text { in } D \times(0, \infty) \\
\nabla \cdot u & =0 \quad \text { in } D \times(0, \infty) \\
u & =0 \quad \text { on } \partial D \times(0, \infty), \\
u(x, 0) & =a(x),
\end{aligned}
$$

where a weak solution $u$ is defined by $u \in L^{\infty}\left(0, T ; L_{\sigma}^{2}(D)\right) \cap L^{2}\left(0, T ; D\left(A^{\frac{1}{2}}\right)\right)$ and

$$
\begin{aligned}
& -\int_{0}^{T}(u(t), v) h^{\prime}(t) d t+\int_{0}^{T}(\nabla u(t), \nabla v) h(t) d t+\int_{0}^{T}((u(t), \nabla) u(t), v) h(t) d t \\
& =h(0)(a, v)+\int_{0}^{T}(f(t), v) h(t) d t
\end{aligned}
$$

for all $v \in D\left(A^{\frac{1}{2}}\right)$ and all $h \in C^{1}([0, T] ; R)$ with $h(T)=0$.

The contents of this paper are as follows: In Sect. 2 we give preliminaries and we set the $3 \mathrm{~d}$ stochastic Navier-Stokes equation (2.1) in a functional analysis setting by using the Stokes operator $A=-P_{2} \Delta$. In Sect. 3 we collect Lemmas used in this paper. In Sect. 4 the existence of a local weak solution to a $3 \mathrm{~d}$ stochastic NS equation with an additive noise is considered following Miyakawa and Sohr [14] and we also consider global existence of solutions. In Sect. 5 the existence of a weak solution to a stochastic Navier-Stokes equation driven by a multiplicative noise is considered. We omit offen the notation $\omega \in \Omega$ if no confusion arises. Let $C$, $c, C_{T}$ and $c_{T}$ denote positive constants which change from a line to a line. 


\section{Preliminaries}

In this paper we use the following Banach spaces:

Let the spaces $C_{0, \sigma}^{\infty}(D)$ and $L_{\sigma}^{q}(D), q>1$ be defined as follows:

$$
C_{0, \sigma}^{\infty}(D):=\text { the space of } \varphi \in C_{0}^{\infty}(D) \text { with } \operatorname{div} \varphi=0 \text { in } D,
$$

$$
L_{\sigma}^{q}(D):=\text { the closure of } C_{0, \sigma}^{\infty}(D) \text { with respect to the } L^{q}(D)-\text { norm }|\cdot|_{q},
$$

where

$$
|u|_{q}=\left(\int_{D}|u|^{q} d x\right)^{\frac{1}{q}} .
$$

Define the forms $B$ and $b$ by

$$
\begin{gathered}
B(u, v)=\sum_{i=1}^{3} u^{i}(x) \frac{\partial v}{\partial x_{i}}(x) d x \\
b(u, v, \varphi)=\int_{D}((u, \nabla) v, \varphi) .
\end{gathered}
$$

We also set

$$
B(u):=B(u, u)
$$

Next let

$$
G^{q}(D):=\left\{\nabla p \in L^{q}(D) ; p \in L_{l o c}^{q}(\bar{D})\right\} .
$$

Then it is well known that the Helmholtz decomposition:

$$
L^{q}(D)=L_{\sigma}^{q}(D) \oplus G^{q}(D)
$$

holds. Let $P_{q}$ denote the projection operator from $L^{q}(D)$ onto $L_{\sigma}^{q}(D)$ and let the operator $A_{q}$ on $L_{\sigma}^{q}(D)$ be defined by $A_{q} u=-P_{q} \Delta u, u \in D\left(A_{q}\right)$ with the domain

$$
D\left(A_{q}\right)=W^{2, q}(D) \cap W_{0}^{1, q}(D) \cap L_{\sigma}^{q}(D) .
$$

The operator $A_{2}$ is a self-adjoint in the real Hilbert space $L_{\sigma}^{2}(D)$. Let $A_{2}=A$. $\left|A^{\frac{1}{2}} u\right|_{2}=|\nabla u|_{2}$ for $u \in D\left(A^{\frac{1}{2}}\right)$. See p.122, [20] in detail.

Let $K, H$ be two separable Hilbert spaces. Let $L(K, H)$ denote the space of all bounded linear operators from $K$ to $H$. Let $Q \in L(K, K)$ be a nonnegative self-adjoint operator. $L_{2}^{0}(K, H)$ denotes the space of all $\xi \in L(K, H)$ such that $\xi \sqrt{Q}$ is a Hilbert-Schmidt operator. The norm is given by

$$
|\xi|_{L_{2}^{0}}^{2}:=|\xi \sqrt{Q}|_{H S}^{2}=\operatorname{tr}\left(\xi Q \xi^{*}\right)<\infty .
$$

Then $\xi$ is called a $Q$-Hilbert-Schmidt operator from $K$ to $H$.

Let $(\Omega, P, \mathcal{F})$ be a complete probability space on which an increasing and right continuous family $\left(\mathcal{F}_{t}\right)_{t \in[0, \infty]}$ of complete sub- $\sigma$-algebra of $\mathcal{F}$ is defined. $\mathcal{F}_{0}$ contains all the null sets of $\mathcal{F}$. Let $\beta_{j}(t)(j=1,2,3, \ldots)$ be a sequence of real valued one-dimensional standard Brownian motions mutually independent on $(\Omega, P, \mathcal{F})$. 
In this paper we set

$$
W(t)=\sum_{n=1}^{\infty} \sqrt{\sigma_{n}} \beta_{n}(t) e_{n}, t \geq 0
$$

where $\sigma_{n} \geq 0(n=1,2,3 \ldots)$ are nonnegative real numbers and $\left\{e_{n}\right\}(n=$ $1,2,3, \ldots)$ is a complete orthonormal basis in the real and separable Hilbert space $K$. Let $Q \in L(K, K)$ be a operator defined by $Q e_{n}=\sigma_{n} e_{n}$. We assume that $Q$ is the trace operator, that is, trace $Q=\Sigma \sigma_{n}<\infty$.

We consider the following stochastic Naver-Stokes equation:

$$
\left\{\begin{array}{c}
d X(t)=[-A X(t)+B(X(t))] d t+g(X(t)) d W(t) \\
X(t, x)=0,(t, x) \in[0, T] \times \Gamma \text { (boundary condition), } \\
X(0)=\phi \text { (initial condition) }
\end{array}\right.
$$

where $\phi$ is an $\mathcal{F}_{0}$-measurable function, $\phi \in D\left(A^{\frac{1}{2}}\right)$ and $g: L_{\sigma}^{2}(D) \rightarrow L_{2}^{0}(K$, $\left.L_{\sigma}^{2}(D)\right)$ is a continuous function. Let $S(t)$ denote an analytic semigroup generated by $-A$.

Definition 1. A stochastic process $X(t)$ is called a mild solution to (2.1) on $[0, T]$, if the following conditions are satisfied:

(a) $X(t)$ is a progressively measurable process such that $X \in L^{\infty}(0, T$; $\left.L_{\sigma}^{2}(D)\right) \cap L^{2}\left(0, T ; D\left(A^{\frac{1}{2}}\right)\right)$, almost surely,

(b) The process $X(t)$ satisfies

$$
X(t)=S(t) \phi+\int_{0}^{t} S(t-s) B(X(s)) d s+\int_{0}^{t} S(t-s) g(X(s)) d W(s)
$$

on $[0, T]$, almost surely.

Definition 2. A stochastic process $X(t)$ is called a weak solution to (2.1) on $[0, T]$, if the following conditions are satisfied:

(c) $X(t)$ is a progressively measurable process such that $X \in L^{\infty}(0, T$; $\left.L_{\sigma}^{2}(D)\right) \cap L^{2}\left(0, T ; D\left(A^{\frac{1}{2}}\right)\right)$, almost surely,

(d) For any fixed $\varphi \in C_{0}^{\infty}(D)$, the process $(X(t), \varphi)$ is continuous,

(e) the process $X(t)$ satisfies

$$
(X(t), \varphi)=\left(X(0)-\int_{0}^{t} A X(s) d s+\int_{0}^{t} B(X(s)) d s+\int_{0}^{t} g(X(s)) d W(s), \varphi\right)
$$

on $[0, T]$, almost surely.

\section{Lemmas}

It is known that $-A_{q}$ generates a uniformly bounded analytic semigroup $\left\{S_{q}(t): t>0\right\}$ on $L_{\sigma}^{q}(D)$. There exists an $M>0$ and a $\beta>0$ such that $|S(t)| \leq M e^{\beta t}, t \geq 0$. 
Lemma 1. The following estimate holds: for $0<\alpha<1$

$$
\left|A_{q}^{\alpha} S_{q}(t)\right|_{q} \leq M_{\alpha q} t^{-\alpha}, M_{\alpha q} \geq 1, t>0 .
$$

Let $M_{\alpha}:=M_{\alpha 2}$. In this paper we use the Yosida approximation

$$
J_{\lambda}:=\left(1+\lambda^{-1} A\right)^{-1} .
$$

Let $\phi_{\lambda}:=J_{\lambda} \phi, f_{\lambda}:=J_{\lambda} f$ and

$$
B_{\lambda}(u, v):=P\left(J_{\lambda} u \cdot \nabla\right) v=B\left(J_{\lambda} u, v\right), B_{\lambda}(u):=B_{\lambda}(u, u) .
$$

Lemma 2. ([14], p. 462) For the Yosida approximation the following hold:

$$
\begin{gathered}
\left|J_{\lambda} w\right|_{2} \leq|w|_{2} \quad \text { for } w \in L_{\sigma}^{2}(D) . \\
\left|J_{\lambda} w-w\right|_{2} \rightarrow 0 \quad \text { as } \lambda \rightarrow \infty \\
\left|J_{\lambda} w\right|_{\infty} \leq C(\lambda)|w|_{2} \quad \text { for } w \in L_{\sigma}^{2}(D) . \\
\left(B_{\lambda}(u, v), w\right)=b\left(J_{\lambda} u, v, w\right) \leq\left|J_{\lambda} u\right|_{\infty}|\nabla v|_{2}|w|_{2} \\
\leq C(\lambda)|u|_{2}|\nabla v|_{2}|w|_{2} .
\end{gathered}
$$

The following lemmas are crucial in this paper. See also Farwig and Komo [9].

Lemma 3. (Theorem 2.7, p. 81, Giga and Sohr [12]). Let $1<q<\infty, 1<r<$ $\infty, 0<T \leq \infty$. Then for every $f \in L^{r}\left(0, T ; L_{\sigma}^{q}(D)\right)$ and $a \in D_{q}^{1-1 / r, r}$ there exists a unique solution of the Stokes equation

$$
u^{\prime}+A_{q} u=f
$$

for a.e. $t \in(0, T), u(0)=a$ satisfying

$$
\int_{0}^{T}\left|u^{\prime}(t)\right|_{q}^{r} d t+\int_{0}^{T}\left|A_{q} u(t)\right|_{q}^{r} d t \leq C\left(\int_{0}^{T}|f(t)|_{q}^{r} d t+|a|_{D_{q}^{1-1 / r, r}}^{r}\right)
$$

where $C=C(q, r, D)>0$ independent of $a, f$ and $T$, and the definition of the space $D_{q}^{1-1 / r, r}$ is given as follows:

$$
D_{q}^{1-1 / r, r}:=\left\{v \in L_{\sigma}^{r}(D) ;|v|_{q}+\left(\int_{0}^{\infty}|A S(t) v|_{q}^{r} d t\right)^{\frac{1}{r}}<\infty\right\} .
$$

Remark 1. The general definition of the space $D_{q}^{\alpha, r}, 0<\alpha<1$, is given in (p.77, [12]).

\section{The existence of a weak solution to (4.2)}

Let $\Phi:[0, T] \times \Omega \rightarrow L_{2}^{0}\left(K, L_{\sigma}^{2}(D)\right)$ be a progressively measurable process. Set

$$
W_{A}(t):=\int_{0}^{t} S(t-s) \Phi(s) d W(s) .
$$


Condition 1. There exist positive constants $\Phi_{2, T}$ and $\Phi_{4, T}>0$ depending on $T>0$ such that

$$
\begin{aligned}
& E\left[\sup _{0 \leq s \leq T}|\Phi(s)|_{L_{0}^{2}}^{2}\right] \vee E\left[\sup _{0 \leq s \leq T}\left|A^{\frac{1}{2}} \Phi(s)\right|_{L_{0}^{2}}^{2}\right]<\Phi_{2, T}, \\
& E\left[\sup _{0 \leq s \leq T}|\Phi(s)|_{L_{0}^{2}}^{4}\right] \vee E\left[\sup _{0 \leq s \leq T}\left|A^{\frac{1}{2}} \Phi(s)\right|_{L_{0}^{2}}^{4}\right]<\Phi_{4, T} .
\end{aligned}
$$

We need the following lemma.

Lemma 4. If, $W_{A}(t)(\omega)$ and $A^{\frac{1}{2}} W_{A}(t)(\omega)$ are continuous, then for a.e. $\omega \in \Omega$, for any given $R_{\omega}>0$ there exists a sufficiently small $T_{0}=T_{0}(\omega)>0$ such that

$$
\sup _{0 \leq t \leq T_{0}}\left|W_{A}(t)(\omega)\right| \vee \sup _{0 \leq t \leq T_{0}}\left|A^{\frac{1}{2}} W_{A}(t)(\omega)\right|<R_{\omega}
$$

In this section we consider the existence of a weak solution to a stochastic Navier-Stokes equation with an additive noise:

$$
\left\{\begin{array}{l}
d X(t)=[-A X(t)+B(X(t))] d t+\Phi(t) d W(t), \\
X(t, x)=0,(t, x) \in[0, \infty) \times \Gamma \text { (boundary condition), } \\
X(0)=\phi \text { (initial condition) }
\end{array}\right.
$$

To this end we first consider the following stochastic equation:

$$
X(t)=S(t) \phi+\int_{0}^{t} S(t-s) B(X(s)) d s+\int_{0}^{t} S(t-s) \Phi(s) d W(s)
$$

for an initial value $\phi$ with $E|\phi|_{2}^{2}<\infty$ and $E\left|A^{\frac{1}{2}} \phi\right|_{2}^{2}<\infty$. Set

$$
Y(t)=X(t)-W_{A}(t) .
$$

Then it follows that

$$
Y(t)=S(t) \phi+\int_{0}^{t} S(t-s) B\left(Y(s)+W_{A}(s)\right) d s .
$$

We consider the following equation

$$
X(t)=S(t) \phi+\int_{0}^{t} S(t-s) B_{\lambda}(X(s)) d s+\int_{0}^{t} S(t-s) \Phi(s) d W(s) .
$$

In other word we ask if there exists a solution $Y(t)(\omega)$ to

$$
Y(t)=S(t) \phi+\int_{0}^{t} S(t-s) B_{\lambda}\left(Y(s)+W_{A}(s)\right) d s, \text { a.e. } \omega \in \Omega .
$$

For any fixed $\phi$ with $E|\phi|_{2}^{2}<\infty$ and $E\left|A^{\frac{1}{2}} \phi\right|_{2}^{2}<\infty$, we can take an $R_{\omega}>0$, for a.e. $\omega \in \Omega$, such that $|\phi(\omega)|_{2}+\left|A^{\frac{1}{2}} \phi(\omega)\right|_{2} \leq \frac{1}{M} R_{\omega}$, where $|S(t)| \leq$ $M, M \geq 1, t \geq 0$. Let $v:[0, T] \times \Omega \rightarrow L_{\sigma}^{2}(D) \cap D\left(A^{\frac{1}{2}}\right)$ and let $\|v(\omega)\|_{T}:=$ $\sup _{0 \leq s \leq T}\left(|v(s)(\omega)|_{2}+\left|A^{\frac{1}{2}} v(s)(\omega)\right|_{2}\right)$. 
Definition 3. Denote by $P P\left([0, T] ; L_{\sigma}^{2}(D) \cap D\left(A^{\frac{1}{2}}\right)\right)$, the set of all $L_{\sigma}^{2}(D) \cap$ $D\left(A^{\frac{1}{2}}\right)$ - valued, $\mathcal{F}_{t}-$ adapted and continuous processes $v(t)$ on $[0, T]$. Then for a.e. $\omega \in \Omega$, define the space

$$
\begin{gathered}
S\left(\phi, T, \omega, R_{\omega}\right) \\
\quad:=\left\{\begin{array}{c}
v \in P P\left([0, T] ; L_{\sigma}^{2}(D) \cap D\left(A^{\frac{1}{2}}\right)\right) \\
v(0)(\omega)=\phi(\omega),\|v(\omega)\|_{T} \leq 2 R_{\omega},|\phi(\omega)|_{2}+\left|A^{\frac{1}{2}} \phi(\omega)\right|_{2} \leq \frac{1}{M} R_{\omega} \cdot
\end{array}\right\}
\end{gathered}
$$

First we show the existence of a solution local in time to (4.5).

Lemma 5. Let $\phi$ be $\mathcal{F}_{0}-$ measurable with $E|\phi|_{2}^{2}<\infty$ and $E\left|A^{\frac{1}{2}} \phi\right|_{2}^{2}<\infty$. Assume that Condition 1 is satisfied. Then there exists a local solution $X_{\lambda}$ to (4.5).

Proof. If Condition 1 is satisfied, then we have that

$$
\begin{aligned}
E & \left|A^{\frac{1}{2}} W_{A}(t)-A^{\frac{1}{2}} W_{A}(\tau)\right|_{2}^{4} \\
= & E\left|\int_{0}^{t} S(t-s) A^{\frac{1}{2}} \Phi(s) d W(s)-\int_{0}^{\tau} S(\tau-s) A^{\frac{1}{2}} \Phi(s) d W(s)\right|_{2}^{4} \\
= & E\left|\int_{\tau}^{t} S(t-s) A^{\frac{1}{2}} \Phi(s) d W(s)+\int_{0}^{\tau}(S(t-s)-S(\tau-s)) A^{\frac{1}{2}} \Phi(s) d W(s)\right|_{2}^{4} \\
\leq & 8 E\left|\int_{\tau}^{t} S(t-s) A^{\frac{1}{2}} \Phi(s) d W(s)\right|_{2}^{4} \\
& +8 E\left|\int_{0}^{\tau}(S(t-s)-S(\tau-s)) A^{\frac{1}{2}} \Phi(s) d W(s)\right|_{2}^{4} .
\end{aligned}
$$

Thus by the Burkholder inequality we obtain that

$$
\begin{aligned}
E \mid & A^{\frac{1}{2}} W_{A}(t)-\left.A^{\frac{1}{2}} W_{A}(\tau)\right|_{2} ^{4} \\
\leq & E\left(\int_{\tau}^{t}\left|S(t-s) A^{\frac{1}{2}} \Phi(s)\right|_{L_{2}^{0}}^{2} d s\right)^{2} \\
& +8 E\left(\int_{0}^{\tau}\left|(S(t-s)-S(\tau-s)) A^{\frac{1}{2}} \Phi(s)\right|_{L_{2}^{0}}^{2} d s\right)^{2} \\
& :=I_{1}+I_{2} .
\end{aligned}
$$

Then

$$
\begin{aligned}
I_{1} & \leq 8 M^{4} \exp (4 \beta T) E\left(\sup _{0 \leq t \leq T}\left|A^{\frac{1}{2}} \Phi(s)\right|_{L_{2}^{0}}^{2} \int_{\tau}^{t} 1 d s\right)^{2} \\
& \leq 24 M^{4} \exp (4 \beta T) \Phi_{4, T}(t-\tau)^{2}
\end{aligned}
$$


Let $\gamma$ be any fixed real number such that $\gamma \in\left(\frac{1}{4}, \frac{1}{2}\right)$. By the similar method as in (p.133 [7])

$$
\begin{aligned}
I_{2} & \leq 8 E\left(\int_{0}^{\tau}\left|\int_{\tau-s}^{t-s} \frac{d}{d \rho} S(\rho) A^{\frac{1}{2}} \Phi(s) d \rho\right|_{L_{2}^{0}}^{2} d s\right)^{2} \\
& \leq 8 E\left(\int_{0}^{\tau}\left|\int_{\tau-s}^{t-s} A S(\rho) A^{\frac{1}{2}} \Phi(s) d \rho\right|_{L_{2}^{0}}^{2} d s\right)^{2} \\
& \leq 8\left(M_{1}^{2} \int_{0}^{\tau}\left|\int_{\tau-s}^{t-s} \frac{d \rho}{\rho}\right|^{2} d s\right)^{2} E\left(\sup \left|A^{\frac{1}{2}} \Phi(s)\right|^{2}\right)^{2} \\
& \leq 8\left(\frac{M_{1}^{2} T^{1-2 \gamma}}{\gamma^{2}(1-2 \gamma)}\right)^{2} \Phi_{4, T}(t-\tau)^{4 \gamma}
\end{aligned}
$$

where we assumed $|t-\tau|<1$. Therefore

$$
E\left|A^{\frac{1}{2}} W_{A}(t)-A^{\frac{1}{2}} W_{A}(\tau)\right|_{2}^{4} \leq c|t-\tau|^{4 \gamma} .
$$

Thus $A^{\frac{1}{2}} W_{A}(t)$ has a continuous modification in $t$ and similarly $W_{A}(t)$ has also a continuous modification in $t$. Let $Z(\omega) \in S\left(\phi, T, \omega, R_{\omega}\right)$ and hence $\|Z(\omega)\|_{T}=\sup _{0 \leq t \leq T}\left(|Z(t)(\omega)|_{2}+\left|A^{\frac{1}{2}} Z(t)(\omega)\right|_{2}\right) \leq 2 R_{\omega}$. Define a mapping $\Gamma$ on the space $S\left(\phi, T, \omega, R_{\omega}\right)$ by

$$
\begin{aligned}
\Gamma(Z) & :=S(t) \phi+\int_{0}^{t} S(t-s) B_{\lambda}\left(Z(s)+W_{A}(s)\right) d s \\
& =S(t) \phi+\int_{0}^{t} S(t-s) B\left(J_{\lambda}\left(Z(s)+W_{A}(s)\right), Z(s)+W_{A}(s)\right) d s .
\end{aligned}
$$

We show that $\Gamma: S\left(\phi, T, \omega, R_{\omega}\right) \rightarrow S\left(\phi, T, \omega, R_{\omega}\right)$ is well defined. By lemmas 1 and 2

$$
\begin{aligned}
& \|\Gamma(Z)\|_{T} \\
& \leq\|S(t) \phi\|_{T}+\left\|\int_{0}^{t} S(t-s) B\left(J_{\lambda}\left(Z(s)+W_{A}(s)\right), Z(s)+W_{A}(s)\right) d s\right\|_{T} \\
& \leq\|S(t) \phi\|_{T}+\sup _{0 \leq t \leq T} \int_{0}^{t}\left|S(t-s) B\left(J_{\lambda}\left(Z(s)+W_{A}(s)\right), Z(s)+W_{A}(s)\right)\right|_{2} d s \\
& \quad+\sup _{0 \leq t \leq T} \int_{0}^{t}\left|A^{\frac{1}{2}} S(t-s) B\left(J_{\lambda}\left(Z(s)+W_{A}(s)\right), Z(s)+W_{A}(s)\right)\right|_{2} d s \\
& \leq\|S(t) \phi\|_{T}+C(\lambda) \sup _{0 \leq t \leq T} \int_{0}^{t}\left|\left(Z(s)+W_{A}(s)\right)\right|_{2}\left|A^{\frac{1}{2}}\left(Z(s)+W_{A}(s)\right)\right|_{2} d s \\
& \quad+C(\lambda) \sup _{0 \leq t \leq T} \int_{0}^{t}\left|A^{\frac{1}{2}} S(t-s)\right|\left|\left(Z(s)+W_{A}(s)\right)\right|_{2} \\
& \quad \times\left|A^{\frac{1}{2}}\left(Z(s)+W_{A}(s)\right)\right|_{2} d s
\end{aligned}
$$




$$
\begin{aligned}
\leq & \sup _{0 \leq t \leq T}\left(|S(t) \phi(\omega)|_{2}+\left|A^{\frac{1}{2}} S(t) \phi(\omega)\right|_{2}\right) \\
& \left.+\left.C(\lambda) \sup _{0 \leq t \leq T} \int_{0}^{t}\left(1+M_{\frac{1}{2}}(t-s)^{-\frac{1}{2}}\right)\left(|Z(s)(\omega)|_{2}+\mid W_{A}(s)\right)(\omega)\right|_{2}\right) \\
& \times\left(\left|A^{\frac{1}{2}} Z(s)(\omega)\right|_{2}+\left|A^{\frac{1}{2}} W_{A}(s)(\omega)\right|_{2}\right) d s .
\end{aligned}
$$

Since thanks to Auxiliary lemma 1 , we have a sufficiently small $T_{0}>0$ such that (4.1) holds and so we can choose a sufficiently small $T \in\left(0, T_{0}\right)>0$ such that

$$
\begin{aligned}
\|\Gamma(Z)(\omega)\|_{T} & \leq R_{\omega}+9 C(\lambda) \sup _{0 \leq t \leq T} \int_{0}^{t}\left(1+M_{\frac{1}{2}}(t-s)^{-\frac{1}{2}}\right) R_{\omega}^{2} d s \\
& \leq R_{\omega}+9 C(\lambda) M_{\frac{1}{2}}(T+2 \sqrt{T}) R_{\omega}^{2} \\
& <2 R_{\omega} .
\end{aligned}
$$

Thus we have that

$$
\Gamma(Z) \in S\left(\phi, T, \omega, R_{\omega}\right) .
$$

Next we show that the mapping $\Gamma$ is a contractive mapping if $T$ is small enough.

$$
\begin{aligned}
& \left\|\Gamma Z_{1}-\Gamma Z_{2}\right\|_{T} \leq \| \int_{0}^{t} S(t-s) B\left(J_{\lambda}\left(Z_{1}(s)-Z_{2}(s)\right), Z_{1}(s)+W_{A}(s)\right) \\
& \quad+S(t-s)\left(B\left(J_{\lambda}\left(Z_{2}(s)+W_{A}(s)\right), Z_{1}(s)-Z_{2}(s)\right)\right) d s \|_{T} \\
& \leq\left\|\int_{0}^{t} S(t-s) B\left(J_{\lambda}\left(Z_{1}(s)-Z_{2}(s)\right), Z_{1}(s)+W_{A}(s)\right) d s\right\|_{T} \\
& \quad+\left\|\int_{0}^{t} S(t-s) B\left(J_{\lambda}\left(Z_{2}(s)+W_{A}(s)\right), Z_{1}(s)-Z_{2}(s)\right) d s\right\|_{T} .
\end{aligned}
$$

Then using Lemmas 2 and 1 we have that

$$
\begin{aligned}
& \int_{0}^{t}|S(t-s)|\left|B\left(J_{\lambda}\left(Z_{1}(s)-Z_{2}(s)\right), Z_{1}(s)+W_{A}(s)\right)\right|_{2} d s \\
& \quad \leq M \int_{0}^{t}\left|J_{\lambda}\left(Z_{1}(s)-Z_{2}(s)\right)\right|_{\infty}\left|A^{\frac{1}{2}}\left(Z_{1}(s)+W_{A}(s)\right)\right|_{2} d s \\
& \quad \leq M C(\lambda) \int_{0}^{t}\left|Z_{1}(s)-Z_{2}(s)\right|_{2}\left|A^{\frac{1}{2}}\left(Z_{1}(s)+W_{A}(s)\right)\right|_{2} d s \\
& \quad \leq 3 M C(\lambda) R_{\omega} \int_{0}^{t}\left|Z_{1}(s)-Z_{2}(s)\right|_{2} d s
\end{aligned}
$$

and

$$
\begin{aligned}
& \int_{0}^{t}\left|A^{\frac{1}{2}} S(t-s)\right|\left|B\left(J_{\lambda}\left(Z_{1}(s)-Z_{2}(s)\right), Z_{1}(s)+W_{A}(s)\right)\right|_{2} d s \\
& \quad \leq M_{\frac{1}{2}} \int_{0}^{t}\left|A^{\frac{1}{2}} S(t-s)\right|\left|J_{\lambda}\left(Z_{1}(s)-Z_{2}(s)\right)\right|_{\infty}\left|A^{\frac{1}{2}}\left(Z_{1}(s)+W_{A}(s)\right)\right|_{2} d s
\end{aligned}
$$




$$
\begin{aligned}
& \leq M_{\frac{1}{2}} C(\lambda) \int_{0}^{t}\left|A^{\frac{1}{2}} S(t-s)\right|\left|Z_{1}(s)-Z_{2}(s)\right|_{2}\left|A^{\frac{1}{2}}\left(Z_{1}(s)+W_{A}(s)\right)\right|_{2} d s \\
& \leq 3 M_{\frac{1}{2}} C(\lambda) R_{\omega} \int_{0}^{t}(t-s)^{-\frac{1}{2}}\left|Z_{1}(s)-Z_{2}(s)\right|_{2} d s .
\end{aligned}
$$

Similarly

$$
\begin{aligned}
& \int_{0}^{t}|S(t-s)|\left|B\left(J_{\lambda}\left(Z_{2}(s)+W_{A}(s)\right), Z_{1}(s)-Z_{2}(s)\right)\right|_{2} d s \\
& \quad+\int_{0}^{t}\left|A^{\frac{1}{2}} S(t-s)\right|\left|B\left(J_{\lambda}\left(Z_{2}(s)+W_{A}(s)\right), Z_{1}(s)-Z_{2}(s)\right)\right|_{2} d s \\
& \quad \leq 3 C_{0} C(\lambda) R_{\omega} \int_{0}^{t}\left(1+(t-s)^{-\frac{1}{2}}\right)\left\|Z_{1}-Z_{2}\right\|_{2} d s,
\end{aligned}
$$

where $C_{0}=\left(M+M_{\frac{1}{2}}\right)$. Therefore

$$
\begin{aligned}
\left\|\Gamma Z_{1}-\Gamma Z_{2}\right\|_{T} & \leq 6 C_{0} C(\lambda) R_{\omega} \sup _{0 \leq t \leq T} \int_{0}^{t}\left(1+(t-s)^{-\frac{1}{2}}\right)\left\|Z_{1}-Z_{2}\right\|_{T} d s \\
& \leq 12 C_{0} C(\lambda) R_{\omega}\left(T+T^{\frac{1}{2}}\right)\left\|Z_{1}-Z_{2}\right\|_{T} .
\end{aligned}
$$

Thus by taking a sufficiently small $T>0$, the mapping $\Gamma$ is a contraction mapping. Thus there exists a fixed point $Y_{\lambda} \in S\left(\phi, T, \omega, R_{\omega}\right)$. Set $X_{\lambda}(s)=$ $Y_{\lambda}(s)+W_{A}(s)$. Then we have that $X_{\lambda}(t)$ satisfies

$$
X_{\lambda}(t)=S(t) \phi+\int_{0}^{t} S(t-s) B_{\lambda}\left(X_{\lambda}(s)\right) d s+\int_{0}^{t} S(t-s) \Phi(s) d W(s) .
$$

Thus the proof of the existence of a local solution is complete.

Next we show the existence of a global weak solution.

Theorem 1. Assume that all the conditions of Lemma 5 and $E|\phi|_{2}^{4}<\infty$ are satisfied. Then there exists a global weak solution $X_{\lambda}(t)$ to (4.5).

Proof. One can prove that a local solution $X_{\lambda}(t)$ to $(4.7)$ is a weak solution by the standard method using the Fubini theorem [2]. That is, $X_{\lambda}(t)$ satisfies for any fixed $\varphi \in C_{0}^{\infty}(D)$,

$$
\left(X_{\lambda}(t), \nabla \varphi\right)=\left(\phi-\int_{0}^{t} A X_{\lambda}(s) d s+\int_{0}^{t} B_{\lambda}\left(X_{\lambda}(s)\right) d s+\int_{0}^{t} \Phi(s) d W(s), \nabla \varphi\right) .
$$

Thus, assume that the solution $X_{\lambda}(s)$ exists on $0 \leq s \leq t \leq t_{0}, 0<t_{0}<T$. Then the proof of the theorem is complete if $E\left|X_{\lambda}\left(t_{0}\right)\right|_{2}^{2}$ and $E\left|A^{\frac{1}{2}} X_{\lambda}\left(t_{0}\right)\right|_{2}^{2}$ are bounded. Since it is clear that $E\left|X_{\lambda}\left(t_{0}\right)\right|_{2}^{2}$ is bounded, we only show that $E\left|A^{\frac{1}{2}} X_{\lambda}\left(t_{0}\right)\right|_{2}^{2}$ is bounded. Applying the Ito formula to $\left|X_{\lambda}(t)\right|_{2}^{4}$, we have that 


$$
\begin{aligned}
& \left|X_{\lambda}(t)\right|_{2}^{4}=|\phi|_{2}^{4}+4 \int_{0}^{t}\left(X_{\lambda}(s)\left|X_{\lambda}(s)\right|_{2}^{2},-A X_{\lambda}(s)+B_{\lambda}\left(X_{\lambda}(s)\right)\right) d s \\
& \quad+6 \int_{0}^{t}|\Phi(s)|_{L_{2}^{0}}^{2}\left|X_{\lambda}(s)\right|_{2}^{2} d s+4 \int_{0}^{t}\left(\left|X_{\lambda}(s)\right|_{2}^{2} X_{\lambda}(s), \Phi(s) d W(s)\right) \\
& \leq|\phi|_{2}^{4}-4 \int_{0}^{t}\left|A^{\frac{1}{2}} X_{\lambda}(s)\right|_{2}^{2}\left|X_{\lambda}(s)\right|_{2}^{2} d s \\
& \quad+3 \int_{0}^{t}|\Phi(s)|_{L_{2}^{0}}^{4}+3 \int_{0}^{t}\left|X_{\lambda}(s)\right|_{2}^{4} d s+4 \int_{0}^{t}\left(\left|X_{\lambda}(s)\right|_{2}^{2} X_{\lambda}(s), \Phi(s) d W(s)\right) .
\end{aligned}
$$

Therefore,

$$
\begin{aligned}
& E\left|X_{\lambda}(t)\right|_{2}^{4}+4 \int_{0}^{t} E\left|A^{\frac{1}{2}} X_{\lambda}(s)\right|_{2}^{2}\left|X_{\lambda}(s)\right|_{2}^{2} d s \\
& \leq E|\phi|_{2}^{4}+3\left[\int_{0}^{t} E|\Phi(s)|_{L_{2}^{0}}^{4} d s+\int_{0}^{t} E\left|X_{\lambda}(s)\right|_{2}^{4} d s\right] .
\end{aligned}
$$

Thus we have a $C_{T}>0$ such that

$$
E\left|X_{\lambda}(t)\right|_{2}^{4}+4 \int_{0}^{t} E\left|A^{\frac{1}{2}} X_{\lambda}(s)\right|_{2}^{2}\left|X_{\lambda}(s)\right|_{2}^{2} d s \leq C_{T} .
$$

By the Ito formula

$$
\begin{aligned}
\left|A^{\frac{1}{2}} X_{\lambda}(t)\right|_{2}^{2}= & \left|A^{\frac{1}{2}} \phi\right|_{2}^{2}-2 \int_{0}^{t}\left|A X_{\lambda}(s)\right|_{2}^{2} d s+2 \int_{0}^{t}\left(A^{\frac{1}{2}} X_{\lambda}(s), A^{\frac{1}{2}} B_{\lambda}\left(X_{\lambda}(s)\right) d s\right. \\
& +\int_{0}^{t}\left|A^{\frac{1}{2}} \Phi(s)\right|_{L_{0}^{2}}^{2} d s+2 \int_{0}^{t}\left(A^{\frac{1}{2}} X_{\lambda}(s), A^{\frac{1}{2}} \Phi(s) d W(s)\right)
\end{aligned}
$$

and hence

$$
\begin{aligned}
E\left|A^{\frac{1}{2}} X_{\lambda}(t)\right|_{2}^{2}+2 \int_{0}^{t} E\left|A X_{\lambda}(t)\right|_{2}^{2} d s \\
\leq E\left|A^{\frac{1}{2}} \phi\right|_{2}^{2}+E \int_{0}^{t}\left|A^{\frac{1}{2}} \Phi(s)\right|_{L_{2}^{0}}^{2} d s \\
\quad+2 E \int_{0}^{t}\left|J_{k} X_{\lambda}(s)\right|_{\infty}\left|\nabla X_{\lambda}(s)\right|_{2}\left|A X_{\lambda}(s)\right|_{2} d s .
\end{aligned}
$$

Then by Lemma 2 and (4.8) we have a $C_{T}>0$ such that

$$
\begin{aligned}
& 2 E \int_{0}^{t}\left|J_{k} X_{\lambda}(s)\right|_{\infty}\left|\nabla X_{\lambda}(s)\right|_{2}\left|A X_{\lambda}(s)\right|_{2} d s \\
& \leq 2 C(\lambda) E \int_{0}^{t}\left|X_{\lambda}(s)\right|_{2}\left|\nabla X_{\lambda}(s)\right|_{2}\left|A X_{\lambda}(s)\right|_{2} d s \\
& \quad \leq E \int_{0}^{t}\left|A X_{\lambda}(s)\right|_{2}^{2} d s+C^{2}(\lambda) E \int_{0}^{t}\left|X_{\lambda}(s)\right|_{2}^{2}\left|A^{\frac{1}{2}} X_{\lambda}(s)\right|_{2}^{2} d s \\
& \leq E \int_{0}^{t}\left|A X_{\lambda}(s)\right|_{2}^{2} d s+C^{2}(\lambda) C_{T} .
\end{aligned}
$$


Thus by Condition 1

$$
\begin{aligned}
& E\left|A^{\frac{1}{2}} X_{\lambda}\left(t_{0}\right)\right|_{2}^{2}+\int_{0}^{t_{0}} E\left|A X_{\lambda}(t)\right|_{2}^{2} d s \\
& \leq E\left|A^{\frac{1}{2}} \phi\right|_{2}^{2}+E \int_{0}^{t_{0}}\left|A^{\frac{1}{2}} \Phi(s)\right|_{L_{2}^{0}}^{2} d s+C^{2}(\lambda) C_{T} \\
& \leq E\left|A^{\frac{1}{2}} \phi\right|_{2}^{2}+T \Phi_{2, T}+C^{2}(\lambda) C_{T} .
\end{aligned}
$$

Thus $E\left|A^{\frac{1}{2}} X_{\lambda}\left(t_{0}\right)\right|_{2}^{2}$ is bounded. This implies that the solution $X_{\lambda}(t)$ exists on $[0, T]$. The proof of the theorem is complete.

\section{Existence of a weak solution to (2.1)}

In this section we first prove the existence of a weak solution $X_{\lambda}$ to

$$
X(t)=\phi-\int_{0}^{t} A X(s) d s+\int_{0}^{t} B_{\lambda}(X(s)) d s+\int_{0}^{t} g(X(s)) d W(s) .
$$

Since a function $g: L_{\sigma}^{2}(D) \rightarrow L_{2}^{0}\left(K, L_{\sigma}^{2}(D)\right)$ is continuous, for any $L_{\sigma}^{2}(D)-$ valued progressively measurable process $\xi$, we have that $g(\xi):[0, T] \times \Omega \rightarrow$ $L_{2}^{0}\left(K, L_{\sigma}^{2}(D)\right)$ is also a progressively measurable process. We need the following condition on $g$.

Condition 2. The function $g: L_{\sigma}^{2}(D) \rightarrow L_{2}^{0}\left(K ; L_{\sigma}^{2}(D)\right)$ satisfies the Lipschitz condition with $g(0)=0$. In other word, there exists an $L_{g}>0$ such that

$$
|g(u)-g(v)|_{L_{2}^{0}}^{2} \leq L_{g}|u-v|_{2}^{2}
$$

for $u, v \in L_{\sigma}^{2}(D)$, and furthermore assume that $g: D\left(A^{\frac{1}{2}}\right) \rightarrow L_{2}^{0}\left(K ; D\left(A^{\frac{1}{2}}\right)\right)$ and there exists a positive constant $L_{G}$ such that

$$
\left|A^{\frac{1}{2}} g(u)-A^{\frac{1}{2}} g(v)\right|_{L_{2}^{0}}^{2} \leq L_{G}\left|A^{\frac{1}{2}} u-A^{\frac{1}{2}} v\right|_{2}^{2}
$$

for $u, v \in D\left(A^{\frac{1}{2}}\right)$.

Condition 3. Assume that an initial value $\phi$ is $\mathcal{F}_{0}-$ measurable and the following conditions are satisfied:

$$
\begin{array}{lll}
E|\phi|_{2}^{2}<\infty & \text { and } & E\left|A^{\frac{1}{2}} \phi\right|_{2}^{2}<\infty . \\
E|\phi|_{2}^{4}<\infty & \text { and } & E\left|A^{\frac{1}{2}} \phi\right|_{2}^{4}<\infty
\end{array}
$$

Assume that $\phi$ satisfies Condition 3 and $g$ satisfies Condition 2. Consider the sequence of the processes.

$$
\left\{\begin{array}{l}
X_{\lambda}^{0}(t):=\phi \\
X_{\lambda}^{n+1}(t)=\phi+\int_{0}^{t}\left[-A X_{\lambda}^{n+1}(s)+B_{\lambda}\left(X_{\lambda}^{n+1}(s)\right)\right] d s \\
\quad+\int_{0}^{t} g\left(X_{\lambda}^{n}(s)\right) d W(s), n \geq 0 .
\end{array}\right.
$$


We have that if the process $X_{\lambda}^{n}(t)(n \geq 1)$ is an $L_{\sigma}^{2}(D)$-progressively measurable process, then $g\left(X_{\lambda}^{n}(t)\right)$ is also a progressively measurable process and so $\int_{0}^{t} g\left(X_{\lambda}^{n}(s)\right) d W(s)$ is well defined. If $E \sup \left|X_{\lambda}^{n}(s)\right|_{2}^{4}$ and $E \sup \left|A^{\frac{1}{2}} X_{\lambda}^{n}(s)\right|_{2}^{2}$ are both bounded, then by Conditions 2 and 3, Theorem 1 and Lemma 7 below the process $X_{\lambda}^{n+1}(t)$ is well defined from (5.2), which is an $\mathcal{F}_{t}-$ measurable and continuous process and hence it is progressively measurable. Thus the sequence $\left\{X_{\lambda}^{n}(t)\right\}(n=1,2,3,,$,$) is well defined.$

Lemma 6. Assume that $a_{0}, a_{1}>0$ and $f^{0}(t):=\beta>0$ are positive constants. Let $f^{n}(t) \geq 0, n \geq 1$. If it holds that for all $n \geq 0$

$$
f^{n+1}(t) \leq a_{0}+a_{1} \int_{0}^{t} f^{n}(s) d s, \quad 0 \leq t \leq T,
$$

then there exists an $M_{\xi}>0$ such that for all $n \geq 0$

$$
f^{n+1}(t) \leq M_{\xi} \exp \left(a_{1} T\right) .
$$

Proof. Let $M_{\xi}:=\max \left\{a_{0}, \beta\right\}$. It holds that $f^{1}(t) \leq M_{\xi}\left(1+a_{1} t\right)$. Assume that the following inequality holds:

$$
f^{n}(t) \leq M_{\xi} \sum_{k=0}^{k=n} \frac{\left(a_{1} t\right)^{k}}{k !}, n \geq 1 .
$$

Then it holds that

$$
\begin{aligned}
f^{n+1}(t) & \leq a_{0}+a_{1} \int_{0}^{t}\left(M_{\xi} \sum_{k=0}^{k=n} \frac{\left(a_{1} s\right)^{k}}{k !}\right) d s \\
& =a_{0}+M_{\xi}\left(\sum_{k=0}^{k=n} \frac{a_{1}^{k+1}(t)^{k+1}}{(k+1) !}\right) \\
& \leq M_{\xi} \sum_{k=0}^{k=n+1} \frac{\left(a_{1} t\right)^{k}}{k !} .
\end{aligned}
$$

This means that it holds that for all $n \geq 0$

$$
f^{n+1}(t) \leq M_{\xi} \exp \left(a_{1} t\right), \quad t \geq 0 .
$$

This completes the proof of the lemma.

Lemma 7. Let $p \geq 2$ and let $\phi$ be $\mathcal{F}_{0}-$ measurable with $E|\phi|_{2}^{p}<\infty$ and $E\left|A^{\frac{1}{2}} \phi\right|_{2}^{2}<\infty$. Assume that Condition 2 is satisfied. Then there exists a constant $B_{e}>0$ such that

$$
E\left[\sup _{0 \leq s \leq T}\left|X_{\lambda}^{n+1}(s)\right|_{2}^{p}\right]<B_{e} \text { and } E\left[\sup _{0 \leq s \leq T}\left|A^{\frac{1}{2}} X_{\lambda}^{n+1}(s)\right|_{2}^{2}\right]<B_{e}
$$

uniformly in all $n \geq 0$. 
Proof. Let $X_{\lambda}^{0}(s)=\phi, 0 \leq s \leq T$. By (5.2), applying the Ito formula to $\left|X_{\lambda}^{n+1}(t)\right|_{2}^{p}$, we have that

$$
\begin{aligned}
& \left|X_{\lambda}^{n+1}(t)\right|_{2}^{p}=|\phi|_{2}^{p} \\
& \quad+p \int_{0}^{t}\left(X_{\lambda}^{n+1}(s)\left|X_{\lambda}^{n+1}(s)\right|_{2}^{p-2},-A X_{\lambda}^{n+1}(s)+B_{\lambda}\left(X_{\lambda}^{n+1}(s)\right)\right) d s \\
& \quad+\frac{1}{2} p(p-1) \int_{0}^{t}\left|g\left(X_{\lambda}^{n}(s)\right)\right|_{L_{2}^{0}}^{2}\left|X_{\lambda}^{n+1}(s)\right|_{2}^{p-2} d s \\
& \quad+p \int_{0}^{t}\left(\left|X_{\lambda}^{n+1}(s)\right|_{2}^{p-2} X_{\lambda}^{n+1}(s), g\left(X_{\lambda}^{n}(s)\right) d W(s)\right) .
\end{aligned}
$$

Therefore,

$$
\begin{aligned}
E & \left.\sup _{0 \leq \tau \leq t}\left|X_{\lambda}^{n+1}(\tau)\right|_{2}^{p}\right]+p \int_{0}^{t} E\left|A^{\frac{1}{2}} X_{\lambda}^{n+1}(s)\right|_{2}^{2}\left|X_{\lambda}^{n+1}(s)\right|_{2}^{p-2} d s \\
\leq & E|\phi|_{2}^{p}+\frac{1}{2} p(p-1) E\left[\int_{0}^{t}\left|g\left(X_{\lambda}^{n}(s)\right)\right|_{L_{2}^{0}}^{2}\left|X_{\lambda}^{n+1}(s)\right|_{2}^{p-2} d s\right] \\
& +p E\left[\sup _{0 \leq \tau \leq t}\left|\int_{0}^{\tau}\left(\left|X_{\lambda}^{n+1}(s)\right|_{2}^{p-2} X_{\lambda}^{n+1}(s), g\left(X_{\lambda}^{n}(s)\right) d W(s)\right)\right|\right] \\
= & E|\phi|_{2}^{p}+J_{1}+J_{2} .
\end{aligned}
$$

Thus we have a constants $p_{1}, p_{2}>0$ such that

$$
\begin{aligned}
J_{1} & \leq \frac{1}{2} p(p-1) L_{g} E\left[\int_{0}^{t}\left|X_{\lambda}^{n}(s)\right|_{2}^{2}\left|X_{\lambda}^{n+1}(s)\right|_{2}^{p-2} d s\right] \\
& \leq p_{1} \int_{0}^{t} E\left|X_{\lambda}^{n}(s)\right|_{2}^{p} d s+p_{2} \int_{0}^{t} E\left|X_{\lambda}^{n+1}(s)\right|_{2}^{p} d s .
\end{aligned}
$$

By the Burkholder-Davis-Gundy lemma, Condition 2 and the Young inequality, there exist positive constants $c, k>0$ such that

$$
\begin{aligned}
J_{2} & \leq 4 c E\left[\left(\int_{0}^{t}\left|X_{\lambda}^{n+1}(s)\right|_{2}^{2(p-2)}\left|X_{\lambda}^{n+1}(s)\right|_{2}^{2}\left|g\left(X_{\lambda}^{n}(s)\right)\right|_{L_{2}^{0}}^{2} d s\right)^{\frac{1}{2}}\right] \\
& \leq 4 c L_{g}^{\frac{1}{2}} E\left[\left(\int_{0}^{t}\left|X_{\lambda}^{n+1}(s)\right|_{2}^{2(p-1)}\left|X_{\lambda}^{n}(s)\right|_{2}^{2} d s\right)^{\frac{1}{2}}\right] \\
& \leq 4 c L_{g}^{\frac{1}{2}} E\left[\sup _{0 \leq s \leq t}\left|X_{\lambda}^{n+1}(s)\right|_{2}^{p-1}\left(\int_{0}^{t}\left|X_{\lambda}^{n}(s)\right|^{2} d s\right)^{\frac{1}{2}}\right] \\
& \leq \frac{1}{2} E\left[\sup _{0 \leq \tau \leq t}\left|X_{\lambda}^{n+1}(\tau)\right|_{2}^{p}\right]+\kappa T^{\frac{p-2}{2}} E\left(\int_{0}^{t}\left|X_{\lambda}^{n}(s)\right|_{2}^{p} d s\right)^{2} .
\end{aligned}
$$


Thus

$$
\begin{aligned}
E\left[\sup _{0 \leq \tau \leq t}\left|X_{\lambda}^{n+1}(\tau)\right|_{2}^{p}\right]+2 p \int_{0}^{t} E\left|A^{\frac{1}{2}} X_{\lambda}^{n+1}(s)\right|_{2}^{2}\left|X_{\lambda}^{n+1}(s)\right|_{2}^{p-2} d s \\
\leq 2 E|\phi|_{2}^{p}+2 p_{1} \int_{0}^{t} E\left|X_{\lambda}^{n}(s)\right|_{2}^{p} d s+2 p_{2} \int_{0}^{t} E\left|X_{\lambda}^{n+1}(s)\right|_{2}^{p} d s \\
\quad+2 \kappa T^{\frac{p-2}{2}} \int_{0}^{t} E\left[\sup _{0 \leq \tau \leq s}\left|X_{\lambda}^{n}(\tau)\right|_{2}^{p}\right] d s
\end{aligned}
$$

By Lemma 6 there exists a constant $B_{c}>0$ such that for all $n \geq 0$

$$
E\left[\sup _{0 \leq s \leq T}\left|X_{\lambda}^{n+1}(s)\right|_{2}^{p}\right]<B_{c}
$$

We also have a constant $C_{T}>0$ such that

$$
\int_{0}^{t} E\left|A^{\frac{1}{2}} X_{\lambda}^{n+1}(s)\right|_{2}^{2}\left|X_{\lambda}^{n+1}(s)\right|_{2}^{2} d s<C_{T}, 0 \leq t \leq T .
$$

Next applying the Ito formula to $\left|A^{\frac{1}{2}} X_{\lambda}^{n+1}(t)\right|_{2}^{2}$, we have that

$$
\begin{aligned}
& \left|A^{\frac{1}{2}} X_{\lambda}^{n+1}(t)\right|_{2}^{2}=\left|A^{\frac{1}{2}} \phi\right|_{2}^{2} \\
& \quad+2 \int_{0}^{t}\left(A^{\frac{1}{2}} X_{\lambda}^{n+1}(s),-A^{\frac{1}{2}} A X_{\lambda}^{n+1}(s)+A^{\frac{1}{2}} B_{\lambda}\left(X_{\lambda}^{n+1}(s)\right)\right) d s \\
& \quad+\int_{0}^{t}\left|A^{\frac{1}{2}} g\left(X_{\lambda}^{n}(s)\right)\right|_{L_{2}^{0}}^{2} d s \\
& \quad+2 \int_{0}^{t}\left(A^{\frac{1}{2}} X_{\lambda}^{n+1}(s), A^{\frac{1}{2}} g\left(X_{\lambda}^{n}(s)\right) d W(s)\right) .
\end{aligned}
$$

Therefore,

$$
\begin{aligned}
E\left[\sup _{0 \leq \tau \leq t}\left|A^{\frac{1}{2}} X_{\lambda}^{n+1}(\tau)\right|_{2}^{2}\right]+2 \int_{0}^{t} E\left|A X_{\lambda}^{n+1}(s)\right|_{2}^{2} d s \\
\leq E\left|A^{\frac{1}{2}} \phi\right|_{2}^{2}+E\left[\int_{0}^{t}\left|A^{\frac{1}{2}} g\left(X_{\lambda}^{n}(s)\right)\right|_{L_{2}^{0}}^{2} d s\right] \\
\quad+2 \int_{0}^{t} E\left|J_{k} X_{\lambda}^{n+1}\right|_{\infty}\left|\nabla X_{\lambda}^{n+1}\right|_{2}\left|A X_{\lambda}^{n+1}\right|_{2} d s \\
\quad+2 E\left[\sup _{0 \leq \tau \leq t}\left|\int_{0}^{\tau}\left(A^{\frac{1}{2}} X_{\lambda}^{n+1}(s), A^{\frac{1}{2}} g\left(X_{\lambda}^{n}(s)\right) d W(s)\right)\right|\right] \\
=E\left|A^{\frac{1}{2}} \phi\right|_{2}^{2}+J_{3}+J_{4}+J_{5} .
\end{aligned}
$$

Thus by Condition 2

$$
J_{3} \leq L_{G} E\left[\int_{0}^{t}\left|A^{\frac{1}{2}} X_{\lambda}^{n}(s)\right|_{2}^{2} d s\right] .
$$


By the Young inequality and (5.3) we have a positive constant $\theta>0$ such that

$$
\begin{aligned}
J_{4} \leq & 2 C(\lambda) \int_{0}^{t} E\left|X_{\lambda}^{n+1}\right|_{2}\left|A^{\frac{1}{2}} X_{\lambda}^{n+1}\right|_{2}\left|A X_{\lambda}^{n+1}\right|_{2} d s \\
\leq & \theta \int_{0}^{t} E\left|X_{\lambda}^{n+1}(s)\right|_{2}^{2}\left|A^{\frac{1}{2}} X_{\lambda}^{n+1}(s)\right|_{2}^{2} d s \\
& +\int_{0}^{t} E\left|A X_{\lambda}^{n+1}(s)\right|_{2}^{2} d s \\
\leq & \theta C_{T}+\int_{0}^{t} E\left|A X_{\lambda}^{n+1}(s)\right|_{2}^{2} d s .
\end{aligned}
$$

By the Burkholder-Davis-Gundy lemma and the Young inequality, there exist constants $c, k>0$ such that

$$
\begin{aligned}
J_{5} & \leq 2 c E\left[\left(\int_{0}^{t}\left|A^{\frac{1}{2}} X_{\lambda}^{n+1}(s)\right|_{2}^{2}\left|A^{\frac{1}{2}} g\left(X_{\lambda}^{n}(s)\right)\right|_{L_{2}^{0}}^{2} d s\right)^{\frac{1}{2}}\right] \\
& \leq 2 c L_{G}^{\frac{1}{2}} E\left[\sup _{0 \leq \tau \leq t}\left|A^{\frac{1}{2}} X_{\lambda}^{n+1}(\tau)\right|_{2}\left(\int_{0}^{t}\left|A^{\frac{1}{2}} X_{\lambda}^{n}(s)\right|_{2}^{2} d s\right)^{\frac{1}{2}}\right] \\
& \leq \frac{1}{2} E\left[\sup _{0 \leq \tau \leq t}\left|A^{\frac{1}{2}} X_{\lambda}^{n+1}(\tau)\right|_{2}^{2}\right]+\kappa E\left(\int_{0}^{t}\left|A^{\frac{1}{2}} X_{\lambda}^{n}(s)\right|_{2}^{2} d s\right) .
\end{aligned}
$$

Thus

$$
\begin{gathered}
E\left[\sup _{0 \leq \tau \leq t}\left|A^{\frac{1}{2}} X_{\lambda}^{n+1}(\tau)\right|_{2}^{2}\right]+2 \int_{0}^{t} E\left|A X_{\lambda}^{n+1}(s)\right|_{2}^{2} d s \\
\leq 2 E\left|A^{\frac{1}{2}} \phi\right|_{2}^{2}+2 L_{G} \int_{0}^{t} E\left[\sup _{0 \leq \tau \leq s}\left|A^{\frac{1}{2}} X_{\lambda}^{n}(\tau)\right|_{2}^{2}\right] d s \\
+2 \kappa \int_{0}^{t} E\left[\sup _{0 \leq \tau \leq s}\left|A^{\frac{1}{2}} X_{\lambda}^{n}(\tau)\right|_{2}^{2}\right] d s+2 \theta C_{T} .
\end{gathered}
$$

By Lemma 6 there exists a constant $B_{d}>0$ such that all $n \geq 0$

$$
E\left[\sup _{0 \leq s \leq T}\left|A^{\frac{1}{2}} X_{\lambda}^{n+1}(s)\right|_{2}^{2}\right]<B_{d}
$$

Thus, the proof of the lemma is complete.

In what follows we prove that there exists a $X_{\lambda}(t)$ such that the sequence $\left\{X_{\lambda}^{n}(t)\right\}$ converges to the $X_{\lambda}(t)$, which is a weak solution to (5.1).

Lemma 8. Assume that Conditions 2 and 3 are satisfied. Then there exists a weak solution $X_{\lambda}(t)$ to $(5.1)$, that is

$$
\left(X_{\lambda}(t), \varphi\right)=\left(\phi+\int_{0}^{t}\left[-A X_{\lambda}(s)+B_{\lambda}\left(X_{\lambda}(s)\right)\right] d s+\int_{0}^{t} g\left(X_{\lambda}(s)\right) d W(s), \varphi\right)
$$


for any $\varphi \in C_{0}^{\infty}(D)$ with $X_{\lambda} \in L^{2}\left(\Omega, C\left(0, T ; L_{\sigma}^{2}(D)\right)\right) \cap L^{2}\left(\Omega \times[0, T] ; D\left(A^{\frac{1}{2}}\right)\right)$. Furthermore $X_{\lambda}(t)$ satisfies that

$$
X_{\lambda}(t)=S(t) \phi+\int_{0}^{t} S(t-s) B_{\lambda}\left(X_{\lambda}(s)\right) d s+\int_{0}^{t} S(t-s) g\left(X_{\lambda}(s)\right) d W(s) .
$$

Proof. From (5.2)

$$
\begin{aligned}
X_{\lambda}^{n+1}(t)-X_{\lambda}^{n}(t)= & \int_{0}^{t}-\left[A X_{\lambda}^{n+1}(s)-A X_{\lambda}^{n}(s)\right] d s \\
& +\int_{0}^{t}\left[B_{\lambda}\left(X_{\lambda}^{n+1}(s)\right)-B_{\lambda}\left(X_{\lambda}^{n}(s)\right)\right] d s \\
& +\int_{0}^{t}\left[g\left(X_{\lambda}^{n}(s)\right)-g\left(X_{\lambda}^{n-1}(s)\right)\right] d W(s) .
\end{aligned}
$$

By applying the Ito formula to $\left|X_{\lambda}^{n+1}(t)-X_{\lambda}^{n}(t)\right|^{2}$,

$$
\left\{\begin{aligned}
\left|X_{\lambda}^{n+1}(t)-X_{\lambda}^{n}(t)\right|_{2}^{2}+\int_{0}^{t}\left|A^{\frac{1}{2}}\left(X_{\lambda}^{n+1}(s)-X_{\lambda}^{n}(s)\right)\right|_{2}^{2} d s \\
=2 \int_{0}^{t}\left(X_{\lambda}^{n+1}(s)-X_{\lambda}^{n}(s), B_{\lambda}\left(X_{\lambda}^{n+1}(s)\right)-B_{\lambda}\left(X_{\lambda}^{n}(s)\right)\right) d s \\
\quad+\int_{0}^{t}\left|g\left(X_{\lambda}^{n}(s)\right)-g\left(X_{\lambda}^{n-1}(s)\right)\right|_{L_{2}^{0}}^{2} d s \\
\quad+2 \int_{0}^{t}\left(X_{\lambda}^{n+1}(s)-X_{\lambda}^{n}(s),\left(g\left(X_{\lambda}^{n}(s)\right)-g\left(X_{\lambda}^{n-1}(s)\right)\right) d W(s)\right) .
\end{aligned}\right.
$$

Let $N$ be any fixed natural integer. Define the stopping time $\tau_{N}$ as follows:

$$
\begin{gathered}
\tau_{N}^{n}:=\inf \left\{0 \leq t \leq T ;\left|X_{\lambda}^{n}(t)\right|_{2} \vee\left|A^{\frac{1}{2}} X_{\lambda}^{n}(s)\right|_{2} \geq N\right\}, \\
\tau_{N}:=\inf _{n \geq N} \tau_{N}^{n} .
\end{gathered}
$$

We have that

$$
\begin{aligned}
E\left[\sup _{0 \leq \tau \leq t \wedge \tau_{N}}\left|X_{\lambda}^{n+1}(\tau)-X_{\lambda}^{n}(\tau)\right|_{2}^{2}\right] \\
\leq 2 E\left[\sup _{0 \leq \tau \leq t \wedge \tau_{N}}\left|\int_{0}^{\tau}\left(X_{\lambda}^{n+1}(s)-X_{\lambda}^{n}(s), B_{\lambda}\left(X_{\lambda}^{n+1}(s)\right)-B_{\lambda}\left(X_{\lambda}^{n}(s)\right)\right) d s\right|\right] \\
\quad+E\left[\sup _{0 \leq \tau \leq t \wedge \tau_{N}}\left|\int_{0}^{\tau}\right| g\left(X_{\lambda}^{n}(s)\right)-\left.g\left(X_{\lambda}^{n-1}(s)\right)\right|_{L_{2}^{0}} ^{2} d s \mid\right] \\
\quad+2 E\left[\sup _{0 \leq \tau \leq t \wedge \tau_{N}}\left|\int_{0}^{\tau}\left(X_{\lambda}^{n+1}(s)-X_{\lambda}^{n}(s),\left(g\left(X_{\lambda}^{n}(s)\right)-g\left(X_{\lambda}^{n-1}(s)\right)\right) d W(s)\right)\right|\right] \\
\quad:=J_{1}+J_{2}+J_{3} .
\end{aligned}
$$

By using Lemma 2, we have that

$$
\begin{aligned}
& \left(B_{\lambda}(u, u)-B_{\lambda}(v, v), u-v\right) \\
& \quad=\left(B\left(J_{\lambda} u-J_{\lambda} v, u\right), u-v\right) \\
& \quad \leq C(\lambda)|\nabla u|_{2}|u-v|_{2}^{2} .
\end{aligned}
$$


Let $n \geq N$. Then

$$
\begin{aligned}
J_{1} & \leq 2 C(\lambda) E \int_{0}^{t}\left|A^{\frac{1}{2}} X_{\lambda}^{n+1}\left(s \wedge \tau_{N}\right)\right|_{2}\left|X_{\lambda}^{n+1}\left(s \wedge \tau_{N}\right)-X_{\lambda}^{n}\left(s \wedge \tau_{N}\right)\right|_{2}^{2} d s \\
& \leq 2 C(\lambda) N \int_{0}^{t} E\left|X_{\lambda}^{n+1}\left(s \wedge \tau_{N}\right)-X_{\lambda}^{n}\left(s \wedge \tau_{N}\right)\right|_{2}^{2} d s .
\end{aligned}
$$

By the Burkholder-Davis-Gundy lemma, we have a $\kappa>0$ such that

$$
\begin{aligned}
J_{3} \leq & \frac{1}{2} E\left[\sup _{0 \leq s \leq t}\left|X_{\lambda}^{n+1}\left(s \wedge \tau_{N}\right)-X_{\lambda}^{n}\left(s \wedge \tau_{N}\right)\right|_{2}^{2}\right] \\
& +\kappa \int_{0}^{t} E\left|g\left(X_{\lambda}^{n}\left(s \wedge \tau_{N}\right)\right)-g\left(X_{\lambda}^{n-1}\left(s \wedge \tau_{N}\right)\right)\right|_{L_{2}^{0}}^{2} d s .
\end{aligned}
$$

Thus by Condition 2 we have a $\gamma>0$ such that for any fixed $n \geq N$,

$$
\left\{\begin{array}{l}
E\left[\sup _{0 \leq s \leq t}\left|X_{\lambda}^{n+1}\left(s \wedge \tau_{N}\right)-X_{\lambda}^{n}\left(s \wedge \tau_{N}\right)\right|_{2}^{2}\right] \\
\quad \leq 4 C(\lambda) N \int_{0}^{t} E\left[\sup _{0 \leq \tau \leq s}\left|X_{\lambda}^{n+1}\left(\tau \wedge \tau_{N}\right)-X_{\lambda}^{n}\left(\tau \wedge \tau_{N}\right)\right|_{2}^{2}\right] d s \\
+\gamma \int_{0}^{t} E \sup _{0 \leq \tau \leq s}\left|X_{\lambda}^{n}\left(\tau \wedge \tau_{N}\right)-X_{\lambda}^{n-1}\left(\tau \wedge \tau_{N}\right)\right|_{2}^{2} d s .
\end{array}\right.
$$

Thus by the Gronwall lemma

$$
\left\{\begin{array}{l}
E\left[\sup _{0 \leq s \leq t}\left|X_{\lambda}^{n+1}\left(s \wedge \tau_{N}\right)-X_{\lambda}^{n}\left(s \wedge \tau_{N}\right)\right|_{2}^{2}\right] \\
\quad \leq \gamma L_{N} \int_{0}^{t} E\left[\sup _{0 \leq \tau \leq s}\left|X_{\lambda}^{n}\left(\tau \wedge \tau_{N}\right)-X_{\lambda}^{n-1}\left(\tau \wedge \tau_{N}\right)\right|_{2}^{2}\right] d s
\end{array}\right.
$$

where $L_{N}:=e^{4 C(\lambda) N T}$. Set

$$
\Psi^{n}(t):=E\left[\sup _{0 \leq s \leq t}\left|X_{\lambda}^{n+1}\left(s \wedge \tau_{N}\right)-X_{\lambda}^{n}\left(s \wedge \tau_{N}\right)\right|_{2}^{2}\right] .
$$

Then we have a $\gamma_{0}>0$ such that

$$
\Psi^{n}(t) \leq \gamma_{0} \int_{0}^{t} \Psi^{n-1}(s) d s, 0 \leq t \leq T .
$$

Thus for any fixed $N>0$, we obtain that the sequence $\left\{X_{\lambda}^{n}\left(t \wedge \tau_{N}\right)\right\}$ is a Cauchy sequence in $L^{2}\left(\Omega, L^{\infty}\left(0, T ; L_{\sigma}^{2}(D)\right)\right)$. Next by the same method from (5.4) we have that the sequence $\left\{X_{\lambda}^{n}\left(t \wedge \tau_{N}\right)\right\}$ is a Cauchy sequence in $L^{2}(\Omega \times$ $\left.[0, T] ; D\left(A^{\frac{1}{2}}\right)\right)$. On the other hand by the Chebyshev inequality and Lemma 7 we have that

$$
P\left(\tau_{N}<T\right)=P\left(\sup _{0 \leq s \leq T}\left|X_{\lambda}^{n}(s)\right|_{2} \vee \sup _{0 \leq s \leq T}\left|A^{\frac{1}{2}} X_{\lambda}^{n}(s)\right|_{2}>N\right) \leq \frac{2 B_{e}}{N^{2}} .
$$

Thus it follows that $P\left(\tau_{N}<T\right) \rightarrow 0$ as $N \rightarrow \infty$. Thus the sequence $\left\{X_{\lambda}^{n}(t)\right\}$ is a Cauchy sequence in $L^{2}\left(\Omega ; L^{\infty}\left(0, T ; L_{\sigma}^{2}(D)\right)\right) \cap L^{2}\left(\Omega \times[0, T] ; D\left(A^{\frac{1}{2}}\right)\right)$. And hence there exists a $X_{\lambda}(t) \in L^{2}\left(\Omega, L^{\infty}\left(0, T ; L_{\sigma}^{2}(D)\right)\right) \cap L^{2}\left(\Omega \times[0, T] ; D\left(A^{\frac{1}{2}}\right)\right)$ such that $X_{\lambda}^{n}(t)$ converges strongly to $X_{\lambda}(t)$. From (5.2) for any fixed $\varphi \in$ 
$C_{0}^{\infty}(D), X_{\lambda}^{n}(t)$ satisfies that

$$
\begin{aligned}
\left(X_{\lambda}^{n+1}(t), \varphi\right)= & \left(\phi+\int_{0}^{t}\left[-A X_{\lambda}^{n+1}(s)+B_{\lambda}\left(X_{\lambda}^{n+1}(s)\right)\right] d s\right. \\
& \left.+\int_{0}^{t} g\left(X_{\lambda}^{n}(s)\right) d W(s), \varphi\right) .
\end{aligned}
$$

Furthermore since

$$
\begin{aligned}
& \left(B_{\lambda}(u, u)-B_{\lambda}(v, v), \varphi\right) \\
& =\left(B\left(J_{\lambda} u-J_{\lambda} v, u\right)+B\left(J_{\lambda} v, u-v\right), \varphi\right) \\
& \quad \leq C(\lambda)|u-v|_{2}|\nabla u|_{2}|\varphi|_{2}+C(\lambda)|u-v|_{2}|v|_{2}|\nabla \varphi|_{2},
\end{aligned}
$$

we have that

$$
\begin{aligned}
E \int_{0}^{t}\left(B_{\lambda}\left(X_{\lambda}^{n+1}(s)\right)-B_{\lambda}\left(X_{\lambda}(s)\right), \varphi\right) d s \\
\leq C(\lambda) E \int_{0}^{t}\left|X_{\lambda}^{n+1}(s)-X_{\lambda}(s)\right|_{2}\left|\nabla X_{\lambda}^{n+1}(s)\right|_{2}|\varphi|_{2} d s \\
\quad+C(\lambda) E \int_{0}^{t}\left|X_{\lambda}^{n+1}(s)-X_{\lambda}(s)\right|_{2}\left|X_{\lambda}(s)\right|_{2}|\nabla \varphi|_{2} d s \\
\leq C(\lambda)\left(E \int_{0}^{t}\left|X_{\lambda}^{n+1}(s)-X_{\lambda}(s)\right|_{2}^{2} d s\right)^{\frac{1}{2}}\left(E \int_{0}^{t}\left|A^{\frac{1}{2}} X_{\lambda}^{n+1}(s)\right|_{2}^{2} d s\right)^{\frac{1}{2}}|\varphi|_{2} \\
\quad+C(\lambda)\left(E \int_{0}^{t}\left|\left(X_{\lambda}^{n+1}(s)-X_{\lambda}(s)\right)\right|_{2}^{2} d s\right)^{\frac{1}{2}}\left(E \int_{0}^{t}\left|X_{\lambda}(s)\right|_{2}^{2} d s\right)^{\frac{1}{2}}|\nabla \varphi|_{2} \\
\rightarrow 0 \quad \text { as } n \rightarrow \infty
\end{aligned}
$$

by the Hölder inequality and Lemma 7 . Therefore the process $X_{\lambda}(t)$ satisfies that for a.e. $\omega \in \Omega$,

$$
\left(X_{\lambda}(t), \varphi\right)=\left(\phi+\int_{0}^{t}\left[-A X_{\lambda}(s)+B_{\lambda}\left(X_{\lambda}(s)\right)\right] d s+\int_{0}^{t} g\left(X_{\lambda}(s)\right) d W(s), \varphi\right)
$$

for $0 \leq t \leq T$. Thus $X_{\lambda}(t)$ is a weak solution to (5.1). The proof of the lemma is complete.

Finally we show that there exists a weak solution $X(t)$ to $(2.1)$.

Theorem 2. Assume that Conditions 2 and 3 are satisfied. Let $E|\phi|_{r}<\infty$ and $E\left|A^{\frac{1}{2}} \phi\right|_{r}<\infty$ with $r=\frac{5}{4}$. Then there exists a weak solution $X(t)$ to (2.1) with

$$
\begin{aligned}
X & \in L^{\infty}\left(0, T ; L_{\sigma}^{2}(D)\right) \cap L^{2}\left(0, T ; D\left(A^{\frac{1}{2}}\right)\right), \\
X \varphi & \in C\left(0, T ; L_{\sigma}^{r}(D)\right) \text { for any fixed } \varphi \in C_{0}^{\infty}(D) .
\end{aligned}
$$


Proof. By lemma 8 there exists a weak solution $X_{\lambda}(t)$ to $(5.1)$. Thus by the Ito formula

$$
\begin{aligned}
\left|X_{\lambda}(t)\right|_{2}^{2}= & |\phi|_{2}^{2}-2 \int_{0}^{t}\left|A^{\frac{1}{2}} X_{\lambda}(s)\right|_{2}^{2} d s \\
& +\int_{0}^{t}\left|g\left(X_{\lambda}(s)\right)\right|_{L_{0}^{2}}^{2} d s+2 \int_{0}^{t}\left(X_{\lambda}(s), g\left(X_{\lambda}(s)\right) d W(s)\right) .
\end{aligned}
$$

By the Burkholder inequality and Condition 2 there exists a $\kappa>0$ such that

$$
\begin{gathered}
\frac{1}{2} E \sup _{0 \leq s \leq t}\left|X_{\lambda}(s)\right|_{2}^{2}+2 E \int_{0}^{t}\left|A^{\frac{1}{2}} X_{\lambda}(s)\right|_{2}^{2} d s \\
\leq E|\phi|_{2}^{2}+(1+\kappa) L_{g} E \int_{0}^{t}\left|X_{\lambda}(s)\right|_{2}^{2} d s .
\end{gathered}
$$

Thus by the Gronwall lemma and Lemma 2 we have that

$$
\begin{gathered}
E \sup _{0 \leq s \leq T}\left|X_{\lambda}(s)\right|_{2}^{2} \leq 2 E|\phi|_{2}^{2} \exp \left(2(1+\kappa) L_{g} T\right), \\
2 E \int_{0}^{T}\left|A^{\frac{1}{2}} X_{\lambda}(s)\right|_{2}^{2} d s \leq E|\phi|_{2}^{2}+M_{\phi} T
\end{gathered}
$$

where $M_{\phi}:=(1+\kappa) L_{g}\left(2 E|\phi|_{2}^{2} \exp \left(2(1+\kappa) L_{g} T\right)\right)$. Since $M_{T}:=$ $2 E|\phi|_{2}^{2} \exp \left(2(1+\kappa) L_{g} T\right)+E|\phi|_{2}^{2}+M_{\phi} T$ are independent of $\lambda$ and

$$
E\left[\sup _{0 \leq s \leq T}\left|X_{\lambda}(s)\right|_{2}^{2}+2 \int_{0}^{T}\left|A^{\frac{1}{2}} X_{\lambda}(s)\right|_{2}^{2} d s\right] \leq M_{T}
$$

we obtain that

$$
P\left(\cup_{M=1}^{\infty} \cap_{j=1}^{\infty} \cup_{\lambda=j}^{\infty}\left\{\sup _{0 \leq s \leq T}\left|X_{\lambda}(s)\right|_{2}^{2}+2 \int_{0}^{T}\left|A^{\frac{1}{2}} X_{\lambda}(s)\right|_{2}^{2} d s \leq M\right\}\right)=1 .
$$

Thus there exist a subsequence $\left\{\alpha_{k}\right\}$ of $\{\lambda\}$ and some constant $M_{\omega}$ for each $\omega \in \Omega_{1}$, where $\Omega_{1}$ is a subset of $\Omega$ with $P\left(\Omega \backslash \Omega_{1}\right)=0$, such that for all $k \geq 1$

$$
\begin{gathered}
{\left[\sup _{0 \leq t \leq T}\left|X_{\alpha_{k}}(t)(\omega)\right|_{2}^{4}\right] \leq M_{\omega}} \\
\int_{0}^{T}\left|A^{\frac{1}{2}} X_{\alpha_{k}}(t)(\omega)\right|_{2}^{2} \leq M_{\omega} .
\end{gathered}
$$

(see $\operatorname{Kim}\left(\right.$ p. 42, [18]). Thus it follows that for each $\omega \in \Omega_{1}$ there exist a $X(\omega)$ and a subsequence $\left\{\lambda_{k}\right\}$ of $\left\{\alpha_{k}\right\}$ such that as $k \rightarrow \infty$,

$$
\begin{gathered}
X_{\lambda_{k}}(\omega) \rightarrow X(\omega) \text { weakly star in } L^{\infty}\left(0, T ; L_{\sigma}^{2}(D)\right), \\
X_{\lambda_{k}}(\omega) \rightarrow X(\omega) \text { weakly in } L^{2}\left(0, T ; D\left(A^{\frac{1}{2}}\right)\right) .
\end{gathered}
$$


Applying the Ito formula to $\left|X_{\lambda_{k}}(t)\right|_{2}^{4}$, we have that

$$
\begin{aligned}
& \left|X_{\lambda_{k}}(t)\right|_{2}^{4}=|\phi|_{2}^{4}+4 \int_{0}^{t}\left(X_{\lambda_{k}}(s)\left|X_{\lambda_{k}}(s)\right|_{2}^{2},-A X_{\lambda_{k}}(s)+B_{\lambda_{k}}\left(X_{\lambda_{k}}(s)\right)\right) d s \\
& \quad+6 \int_{0}^{t}\left|g\left(X_{\lambda_{k}}(s)\right)\right|_{L_{2}^{0}}^{2}\left|X_{\lambda_{k}}(s)\right|_{2}^{2} d s \\
& \quad+4 \int_{0}^{t}\left(\left|X_{\lambda_{k}}(s)\right|_{2}^{2} X_{\lambda_{k}}(s), g\left(X_{\lambda_{k}}(s)\right) d W(s)\right) \\
& \leq|\phi|_{2}^{4}-4 \int_{0}^{t}\left|A^{\frac{1}{2}} X_{\lambda_{k}}(s)\right|_{2}^{2}\left|X_{\lambda_{k}}(s)\right|_{2}^{2} d s \\
& \quad+6 L_{g} \int_{0}^{t}\left|X_{\lambda_{k}}(s)\right|_{2}^{4} d s+4 \int_{0}^{t}\left(\left|X_{\lambda_{k}}(s)\right|_{2}^{2} X_{\lambda_{k}}(s), g\left(X_{\lambda_{k}}(s)\right) d W(s)\right) .
\end{aligned}
$$

Therefore, by the Burkfolder inequality and the Young inequality, there exists a positive constant $c_{T}$ independent of $\lambda_{k}$ such that

$$
\begin{aligned}
& \frac{1}{2} E \sup _{0 \leq \tau \leq t}\left|X_{\lambda_{k}}(\tau)\right|_{2}^{4}+4 \int_{0}^{t} E\left|A^{\frac{1}{2}} X_{\lambda_{k}}(s)\right|_{2}^{2}\left|X_{\lambda_{k}}(s)\right|_{2}^{2} d s \\
& \quad \leq 2 E|\phi|_{2}^{4}+c_{T} \int_{0}^{t} E \sup _{0 \leq \tau \leq s}\left|X_{\lambda_{k}}(\tau)\right|_{2}^{4} d s
\end{aligned}
$$

Thus by the Gronwall lemma we have that

$$
E \sup _{0 \leq t \leq T}\left|X_{\lambda_{k}}(t)\right|_{2}^{4} \leq 4 E|\phi|_{2}^{4} \exp \left(2 c_{T} T\right)
$$

Let

$$
Y_{\lambda_{k}}(t):=X_{\lambda_{k}}(t)-\int_{0}^{t} S(t-s) g\left(X_{\lambda_{k}}(s)\right) d W(s)
$$

Then

$$
Y_{\lambda_{k}}(t)=S(t) \phi+\int_{0}^{t} S(t-s) B_{\lambda_{k}}\left(X_{\lambda_{k}}(s)\right) d s .
$$

It follows that

$$
\begin{aligned}
E \int_{0}^{T}\left|A^{\frac{1}{2}} Y_{\lambda_{k}}(t)\right|_{2}^{2} d t \leq 2 E \int_{0}^{T}\left|A^{\frac{1}{2}} X_{\lambda_{k}}(t)\right|_{2}^{2} d t \\
\quad+2 E \int_{0}^{T}\left|\int_{0}^{t} S(t-s) A^{\frac{1}{2}} g\left(X_{\lambda_{k}}(s)\right) d W(s)\right|_{2}^{2} d t \\
\leq 2 E \int_{0}^{T}\left|A^{\frac{1}{2}} X_{\lambda_{k}}(t)\right|_{2}^{2} d t \\
\quad+2 M^{2} E \int_{0}^{T}\left(\int_{0}^{t}\left|A^{\frac{1}{2}} g\left(X_{\lambda_{k}}(s)\right)\right|_{L_{2}^{0}}^{2} d s\right) d t \\
\leq 2 E \int_{0}^{T}\left|A^{\frac{1}{2}} X_{\lambda_{k}}(t)\right|_{2}^{2} d t+2 M^{2} L_{G} T E \int_{0}^{T}\left|A^{\frac{1}{2}} X_{\lambda_{k}}(s)\right|_{2}^{2} d s
\end{aligned}
$$


Thus we have a $C_{T}>0$ such that

$$
E \int_{0}^{T}\left|A^{\frac{1}{2}} Y_{\lambda_{k}}(t)\right|_{2}^{2} d t \leq C_{T}
$$

Next we have a constant $\hat{c}_{T}>0$ such that

$$
\begin{aligned}
E\left[\sup _{0 \leq t \leq T}\left|Y_{\lambda_{k}}(t)\right|_{2}^{4}\right] \leq & 8 E\left[\sup _{0 \leq t \leq T}\left|X_{\lambda_{k}}(t)\right|_{2}^{4}\right] \\
& +8 E\left[\sup _{0 \leq t \leq T}\left|\int_{0}^{t} S(t-s) g\left(X_{\lambda_{k}}(s)\right) d W(s)\right|_{2}^{4}\right] \\
\leq & 32 E|\phi|_{2}^{4} \exp \left(2 c_{T} T\right)+8 \hat{c}_{T} E \int_{0}^{T}\left|g\left(X_{\lambda_{k}}(s)\right)\right|_{L_{2}^{0}}^{4} d s \\
\leq & C_{T}
\end{aligned}
$$

where $C_{T}:=32 E|\phi|_{2}^{4} \exp \left(2 c_{T} T\right)\left(1+\hat{c}_{T} L_{g}^{2} T\right)$. Thus we have that

$$
P\left(\cup_{N=1}^{\infty} \cap_{j=1}^{\infty} \cup_{k=j}^{\infty}\left\{\sup _{0 \leq t \leq T}\left|Y_{\lambda_{k}}(t)\right|_{2}^{4}+\int_{0}^{T}\left|A^{\frac{1}{2}} Y_{\lambda_{k}}(t)(\omega)\right|_{2}^{2} \leq N\right\}\right)=1 .
$$

And hence there exist some subsequence $\left\{\hat{\theta}_{k}\right\}$ of $\lambda_{k}$ and some constant $N_{\omega}>0$ for each $\omega \in \Omega_{2}$, where $\Omega_{2}$ is a subset of $\Omega_{1}$ with $P\left(\Omega \backslash \Omega_{2}\right)=0$, such that for all $k \geq 1$

$$
\begin{gathered}
{\left[\sup _{0 \leq t \leq T}\left|Y_{\hat{\theta}_{k}}(t)(\omega)\right|_{2}^{4}\right] \leq N_{\omega},} \\
\int_{0}^{T}\left|A^{\frac{1}{2}} Y_{\hat{\theta}_{k}}(t)(\omega)\right|_{2}^{2} \leq N_{\omega}
\end{gathered}
$$

since $C_{T}$ is independent of $\lambda_{k}$. And hence it follows that there exist a $Y(t)(\omega) \in$ $L^{\infty}\left(0, T ; L_{\sigma}^{2}(D)\right) \cap L^{2}\left(0, T ; D\left(A^{\frac{1}{2}}\right)\right)$ and a subsequence $\left\{\theta_{k}\right\}$ of $\left\{\hat{\theta}_{k}\right\}$ such that

$$
\begin{gathered}
Y_{\theta_{k}}(t)(\omega) \rightarrow Y(t)(\omega) \text { weakly star in } L^{\infty}\left(0, T ; L_{\sigma}^{2}(D)\right), \\
Y_{\theta_{k}}(t)(\omega) \rightarrow Y(t)(\omega) \text { weakly in } L^{2}\left(0, T ; D\left(A^{\frac{1}{2}}\right)\right) .
\end{gathered}
$$

Let $n=3, r=\frac{n+2}{n+1}, \frac{1}{2^{*}}=\frac{1}{2}-\frac{1}{n}$ and $q=\frac{2(n+2)}{n}$. Then by the Hölder inequality, Lemma 2 and the Interpolation lemma,

$$
\begin{aligned}
\left|B_{\theta_{k}}\left(X_{\theta_{k}}(t)(\omega)\right)\right|_{r} & \leq\left|J_{\theta_{k}} X_{\theta_{k}}(t)(\omega)\right|_{q}\left|A^{\frac{1}{2}} X_{\theta_{k}}(t)(\omega)\right|_{2} \\
& \leq C\left|J_{\theta_{k}} X_{\theta_{k}}(t)(\omega)\right|_{2}^{\frac{2}{n+2}}\left|J_{\theta_{k}} X_{\theta_{k}}(t)(\omega)\right|_{2^{*}}^{\frac{n}{n+2}}\left|A^{\frac{1}{2}} X_{\theta_{k}}(t)(\omega)\right|_{2} \\
& \leq C\left|X_{\theta_{k}}(t)(\omega)\right|_{2}^{\frac{2}{n+2}}\left|A^{\frac{1}{2}} X_{\theta_{k}}(t)(\omega)\right|_{2}^{\frac{2}{r}},
\end{aligned}
$$


where we used Lemma 3.3 [14]. Since by (5.8) and (5.9) for each $\omega \in \Omega_{2}$, there exists a $c_{0}(\omega)>0$ such that

$$
\begin{aligned}
\int_{0}^{T}\left|B_{\theta_{k}}\left(X_{\theta_{k}}(t)(\omega)\right)\right|_{r}^{r} d t & \leq C \int_{0}^{T}\left|X_{\theta_{k}}(t)(\omega)\right|_{2}^{\frac{2 r}{n+2}}\left|A^{\frac{1}{2}} X_{\theta_{k}}(t)(\omega)\right|_{2}^{2} d t \\
& <c_{0}(\omega) .
\end{aligned}
$$

Since $E|\phi|_{\frac{5}{4}}, E\left|A^{\frac{1}{2}} \phi\right|_{\frac{5}{4}}<\infty$, there exists an $a_{\omega}>0$ for each $\omega \in \Omega_{2}$ such that $|\phi(\omega)|_{\frac{5}{4}}<a_{\omega}$ and $\left|A^{\frac{1}{2}} \phi(\omega)\right|_{\frac{5}{4}}<a_{\omega}$. Furthermore

$$
\begin{aligned}
\int_{0}^{\infty}|A S(t) \phi(\omega)|_{r}^{r} d t & \leq \int_{0}^{1}|A S(t) \phi(\omega)|_{r}^{r} d t+\int_{1}^{\infty}|A S(t) \phi(\omega)|_{r}^{r} d t \\
& \leq \int_{0}^{1}\left|A^{\frac{1}{2}} S(t)\right|^{r}\left|A^{\frac{1}{2}} \phi(\omega)\right|_{r}^{r} d t+\int_{1}^{\infty}|A S(t)|^{r}|\phi(\omega)|_{r}^{r} d t \\
& \leq M_{\frac{1}{2}}^{r} \int_{0}^{1} t^{-\frac{r}{2}} d t\left|A^{\frac{1}{2}} \phi(\omega)\right|_{r}^{r}+M_{1}^{r} \int_{1}^{\infty} t^{-r}|\phi(\omega)|_{r}^{r} d t \\
& \leq \frac{8 M_{\frac{1}{2}}^{r}\left|A^{\frac{1}{2}} \phi(\omega)\right|_{r}^{r}}{3}+4 M_{1}^{r}|\phi(\omega)|_{r}^{r} \\
& <\infty, \quad r=\frac{5}{4} .
\end{aligned}
$$

From (5.12), by Lemma 3, for a.e. $\omega \in \Omega$ (we use this presentation notation if no cofusion arises) and each $\theta_{k}>0$,

$$
\frac{d}{d t} Y_{\theta_{k}}(t)(\omega)+A_{r} Y_{\theta_{k}}(t)(\omega)=B_{\theta_{k}}\left(X_{\theta_{k}}(t)(\omega)\right) .
$$

Thus from Lemma 3, there exists a $C_{T}(\omega)>0$ such that

$$
\int_{0}^{T}\left|\frac{d}{d t} Y_{\theta_{k}}(t)(\omega)\right|_{r}^{r} d t<C_{T}(\omega)
$$

Thus for any fixed $\varphi \in C_{0}^{\infty}(D)$,

$$
\int_{0}^{T}\left|\frac{d}{d t}\left(Y_{\theta_{k}}(t)(\omega) \varphi\right)\right|_{r}^{r} d t<|\varphi|_{\infty}^{r} C_{T}(\omega) .
$$

And let $B_{\delta}$ be a closed ball with a radius $\delta$ in $C\left([0, T] ; L_{\sigma}^{r}(K(\varphi))\right.$. Then $\left\{Y(t) \in B_{\delta}\right\} \cap \Omega_{1}$ is $\mathcal{F}_{t}$-adapted since it holds that

$$
\begin{aligned}
& \left\{Y(t) \in B_{\delta}\right\} \cap \Omega_{1} \\
& \quad=\Omega_{1} \cap \cup_{L=1}^{\infty} \cap_{\nu=1}^{\infty} \cap_{j=1}^{\infty} \cup_{k=j}^{\infty}\left(\left(Y_{\theta_{k}}(t) \in B_{\delta+\frac{1}{\nu}}\right) \cap \sup _{0 \leq s \leq t}\left|Y_{\theta_{k}}(s)\right| \leq L\right),
\end{aligned}
$$

where let $\Omega_{1}$ be a subset of $\Omega$ with $P\left(\Omega \backslash \Omega_{1}\right)=0$. Thus by Proposition 1.13 [15], for every $B_{\alpha} \in \operatorname{Borel}\left(L_{\sigma}^{2}(D)\right)$,

$$
\left\{(s, \omega): 0 \leq s \leq t,\left(Y(s, \omega) \in B_{\alpha}\right\} \in B([0, t]) \times \mathcal{F}_{t} .\right.
$$


Since every closed ball of finite radius in $L_{\sigma}^{2}(K(\varphi))$ is closed in $L_{\sigma}^{r}(K(\varphi))$, it follows that $Y(\omega) \varphi$ is an $L_{\sigma}^{2}(K(\varphi))$-valued progressively measurable. By (5.18) and (5.19) it holds that $Y(t)(\omega) \varphi \in L^{\infty}\left(0, T ; L_{\sigma}^{2}(K(\varphi)) \cap L^{2}\left(0, T ; D\left(A^{\frac{1}{2}}\right)\right)\right.$ and

$$
\begin{gathered}
Y_{\theta_{k}}(t)(\omega) \varphi \rightarrow Y(t)(\omega) \varphi \text { weakly star in } L^{\infty}\left(0, T ; L_{\sigma}^{2}(K(\varphi)),\right. \\
Y_{\theta_{k}}(t)(\omega) \varphi \rightarrow Y(t)(\omega) \varphi \text { weakly in } L^{2}\left(0, T ; D\left(A^{\frac{1}{2}}\right)\right)
\end{gathered}
$$

as $k \rightarrow \infty$. Since

$$
D\left(A^{\frac{1}{2}}\right) \cap L_{\sigma}^{2}(K(\varphi)) \subset L_{\sigma}^{2}(K(\varphi)) \subset L_{\sigma}^{r}(K(\varphi)),
$$

by using the compactness lemma (Theorem 2.1, [14]) and by (5.16) for a.e.w $\in$ $\Omega$, there exists a subsequence $\left\{\xi_{i}\right\}$ of $\left\{\rho_{k}\right\}$ such that

$$
Y_{\xi_{j}}(t)(\omega) \varphi \rightarrow Y(t)(\omega) \varphi \text { strongly in } L^{2}\left(0, T ; L_{\sigma}^{2}(K(\varphi))\right),
$$

as $j \rightarrow \infty$. We have that

$$
\begin{aligned}
& E\left|X_{\xi_{j}}(t) \varphi-X_{\xi_{j+1}}(t) \varphi\right|_{2}^{2} \\
& \leq E \mid\left(Y_{\xi_{j}}(t) \varphi+\varphi \int_{0}^{t} S(t-s) g\left(X_{\xi_{j}}(s)\right) d W(s)\right) \\
&-\left.\left(Y_{\xi_{j+1}}(t) \varphi+\varphi \int_{0}^{t} S(t-s) g\left(X_{\xi_{j+1}}(s)\right) d W(s)\right)\right|_{2} ^{2} \\
& \leq 2 E\left|Y_{\xi_{j}}(t) \varphi-Y_{\xi_{j+1}}(t) \varphi\right|_{2}^{2} \\
&+2 E\left|\varphi \int_{0}^{t} S(t-s) g\left(X_{\xi_{j}}(s)\right) d W(s)-\varphi \int_{0}^{t} S(t-s) g\left(X_{\xi_{j+1}}(s)\right) d W(s)\right|_{2}^{2} \\
& \leq 2 E\left|Y_{\xi_{j}}(t) \varphi-Y_{\xi_{j+1}}(t) \varphi\right|_{2}^{2}+2 M^{2} C L_{g}^{2} \int_{0}^{t} E\left|X_{\xi_{j}}(s) \varphi-X_{\xi_{j+1}}(s) \varphi\right|_{2}^{2} d s .
\end{aligned}
$$

Thus by the Gronwall lemma it follows that

$$
\begin{aligned}
& E \int_{0}^{T}\left|X_{\xi_{j}}(t) \varphi-X_{\xi_{j+1}}(t) \varphi\right|_{2}^{2} d t \\
& \quad \leq 2 E \int_{0}^{T}\left|Y_{\xi_{j}}(t) \varphi-Y_{\xi_{j+1}}(t) \varphi\right|_{2}^{2} d t \exp \left(2 M^{2} C L_{g}^{2} T\right) \\
& \quad \rightarrow 0 \quad \text { as } j \rightarrow \infty .
\end{aligned}
$$

by using (5.21). It follows that the sequence $\left\{X_{\xi_{j}}(t)(\omega) \varphi\right\}$ is a Cauchy sequence in mean square. Thus there exists a subsequence $\left\{\rho_{k}\right\}$ of $\left\{\xi_{j}\right\}$ such that $X_{\rho_{k}}(t)(\omega) \varphi$ converges strongly to $X(t)(\omega) \varphi$ in $L^{2}\left(0, T ; L_{\sigma}^{2}(D)\right)$ as $k \rightarrow \infty$ since $\left\{X_{\xi_{j}}(t)(\omega) \varphi\right\}$ has a subsequence which converges weakly to $X(t)(\omega) \varphi$ 
by (5.10) and (5.11). We use the notation $\rho$ instead of $\rho_{k}$ for the simplicity.

$$
\begin{aligned}
& \left|\int_{0}^{t}\left(B_{\rho}\left(X_{\rho}(s)(\omega)\right)-B(X(s)(\omega)), \varphi\right) d s\right| \\
& \quad \leq \int_{0}^{t}\left|b\left(J_{\rho} X_{\rho}(s)(\omega)-X(s)(\omega), X_{\rho}(s)(\omega), \varphi\right)\right| d s \\
& \quad+\int_{0}^{t}\left|b\left(X(s)(\omega), X_{\rho}(s)(\omega)-X(s)(\omega), \varphi\right)\right| d s \\
& \quad:=I_{1}+I_{2},
\end{aligned}
$$

and hence it follows that for a.e. $\omega \in \Omega$,

$$
\begin{aligned}
I_{1} & \leq \int_{0}^{t} \int_{K(\varphi)} \mid \varphi\left(J_{\rho} X_{\rho}(s)(\omega)-X(s)(\omega)|| \nabla X_{\rho}(s)(\omega) \mid d s\right. \\
& \leq|\varphi|_{\infty} \int_{0}^{t}\left|J_{\rho} X_{\rho}(s)(\omega)-X(s)(\omega)\right|_{2, K(\varphi)}\left|\nabla X_{\rho}(s)(\omega)\right|_{2, K(\varphi)} d s \\
& \leq|\varphi|_{\infty}\left(\int_{0}^{t}\left|J_{\rho} X_{\rho}(s)(\omega)-X(s)(\omega)\right|_{2, K(\varphi)}^{2}\right)^{\frac{1}{2}}\left(\int_{0}^{t}\left|\nabla X_{\rho}(s)(\omega)\right|_{2, K(\varphi)}^{2} d s\right)^{\frac{1}{2}} \\
& \rightarrow 0 \text { as } \rho \rightarrow \infty,
\end{aligned}
$$

where $|f|_{2, K(\varphi)}^{2}:=\int_{K(\varphi)}|f(x)|^{2} d x$ and we used that

$$
\begin{aligned}
\left|J_{\rho} X_{\rho}(s)-X(s)\right|_{2, K(\varphi)} & \leq\left|J_{\rho} X_{\rho}(s)-J_{\rho} X(s)\right|_{2, K(\varphi)}+\left|J_{\rho} X(s)-X(s)\right|_{2, K(\varphi)} \\
& \leq\left|X_{\rho}(s)-X(s)\right|_{2, K(\varphi)}+\left|J_{\rho} X(s)-X(s)\right|_{2, K(\varphi)}
\end{aligned}
$$

and $\left|\nabla X_{\rho}(s)(\omega)\right|_{2}=\left|A^{\frac{1}{2}} X_{\rho}(s)(\omega)\right|_{2}(\operatorname{see}[13])$. Then we can choose a a suitable subsequence $\rho_{i}$ of $\rho$. Let $\xi(x)$ be a positive function in $C_{0}^{\infty}$ such that $K(\varphi) \subset$ $K(\xi)$ and $\xi=1$ on $K(\varphi)$, where $K(\varphi)$ and $K(\xi)$ denote the compact support of $\varphi$ and $\xi$, respectively. We have that

$$
\begin{aligned}
\left|\left(X_{\rho_{i}}(s)(\omega)-X(s)(\omega)\right)\right|_{2, K(\varphi)} & \leq\left|\left(X_{\rho_{i}}(s)(\omega) \xi-X(s)(\omega) \xi\right)\right|_{2, K(\xi)} \\
& \rightarrow 0 \quad \text { as } i \rightarrow \infty .
\end{aligned}
$$

Hence

$$
\begin{aligned}
I_{2} & \leq \int_{0}^{t}\left|\int_{K(\varphi)} \nabla(\varphi X(s)(\omega))\left(X_{\rho}(s)(\omega)-X(s)(\omega)\right) d x\right| d s \\
& \leq \int_{0}^{t}\left(\int_{K(\varphi)}|\nabla(\varphi X(s)(\omega))|^{2}\right)^{\frac{1}{2}}\left|\left(X_{\rho_{i}}(s)(\omega)-X(s)(\omega)\right)\right|_{2, K(\varphi)} d s \\
& \leq c \int_{0}^{t}\left|\left(X_{\rho_{i}}(s)(\omega)-X(s)(\omega)\right)\right|_{2, K(\varphi)} d s \rightarrow 0 \text { as } i \rightarrow \infty .
\end{aligned}
$$

Thus we have that

$$
\left|\int_{0}^{t}\left(B_{\rho_{i}}\left(X_{\rho}(s)(\omega)\right)-B(X(s)(\omega)), \varphi\right) d s\right| \rightarrow 0
$$

as $i \rightarrow \infty$. Next we assume that $\varphi \geq 0$ without loss of generality 


$$
\begin{aligned}
E & \left|\int_{0}^{t}\left(g\left(X_{\rho}(s)\right)-g(X(s)), \varphi\right) d W(s)\right| \\
& =E\left|\int_{0}^{t}\left(\left(g\left(X_{\rho}(s)\right)-g(X(s))\right) d W(s), \varphi\right)\right| \\
& \leq c\left(\int_{0}^{t} E\left|\varphi g\left(X_{\rho}(s)\right)-\varphi g(X(s))\right|_{L_{2}^{0}}^{2} d s\right)^{\frac{1}{2}} \\
& \leq c L_{g}^{\frac{1}{2}}\left(\int_{0}^{t} E \varphi\left|X_{\rho}(s)-X(s)\right|_{2}^{2} d s\right)^{\frac{1}{2}} \\
& \leq c L_{g}^{\frac{1}{2}}\left(E \int_{0}^{t}\left|\varphi X_{\rho}(s)-\varphi X(s)\right|_{2}^{2} d s\right)^{\frac{1}{2}} \\
& \rightarrow 0 .
\end{aligned}
$$

Thus we obtain that almost surely

$$
(X(t), \varphi)=\left(\phi-\int_{0}^{t} A X(s) d s+\int_{0}^{t} B(X(s)) d s+\int_{0}^{t} g(X(s)) d W(s), \varphi\right) .
$$

Consequently $X(t)$ is a weak solution to (2.1). This completes the proof of the theorem.

\section{Acknowledgements}

The author would like to strongly appreciate to referees for their kind comments and nice advises. This research is partly supported by the Grant-in-Aid for Scientific Research (N0. 24540198) from Japan Society for the Promotion of Science (JSPS), Japan.

\section{References}

[1] Brzezniak, Z., Li, Y.: Asymptotic compactness and absorbing sets of 2D stochastic Navier-Stokes equations on some unbounded domains. Trans. Am. Math. Soc. 358, 5587-5629 (2006)

[2] Brzezniak, Z., Motyl, E.: Existence of a martingale solution to the stochastic Boussinesq equations. Glob. Stoch. 1, 175-216 (2011)

[3] Brzezniak, Z., Motyl, E.: The existence of martingale solutions to the stochastic Navier-Stokes equations in unbounded 2D and 3D-domains. J. Differ. Equ. 254, 1627-1685 (2013)

[4] Brzezniak, Z., Peszat, S.: Strong local and global solutions for stochastic NavierStokes equations. In: Infinite Dimensional Stochastic Analysis, pp. 85-98, North Holland, Amsterdam (1999)

[5] Capinski, M., Gatarek, D.: Stochastic equations in Hilbert space with application to Navier-Stokes equations in any dimension. J. Funct. Anal. 126, 26-35 (1994) 
[6] Capinski, M., Peszat, S.: On the existence of a solution to stochastic NavierStokes equations. Nonlinear Anal. 44, 141-177 (2001)

[7] Da Prato, G., Zabczyk, J.: Stochastic Equations in Infinite Dimensions. Cambridge University Press, Cambridge (1992)

[8] Da Prato, G.; Zabczyk, J.: Ergodicity for Infinite Dimensional Systems. Cambridge University Press, Cambridge (1996)

[9] Farwig, R., Komo, C.: Regularity of weak solutions to the Navier-Stokes equations in exterior domains. Nonlinear Diff. Equ. Appl. 17, 303-321 (2010)

[10] Flandoli, F., Gatarek, D.: Martingale and stationary solutions for stochastic Naver-Stokes equations. Proba. Theory Relat. Fields 102, 365-391 (1995)

[11] Flandoli, F.: An introduction to 3D stochastic fluid dynamics, Lecture notes, vol. 1942 , pp. 51-150 (2008)

[12] Giga, Y., Sohr, H.: Abstract $L^{p}$ estimates for the Cauchy probulem with applications to the Navier-Stokes equations in exterior Domains. J. Funct. Anal. 102, 72-94 (1991)

[13] Fujita, H., Kato, T.: On the Navier-Stokes initial value problem 1. Arch. Ration. Mech. Anal. 16, 269-315 (1964)

[14] Miyakawa, T., Sohr, H.: On energy inequality, smoothness and large time behavior in $L^{2}$ for weak solutions of the Navier-Stokes equations in exterior domains. Math. Z 199, 455-478 (1988)

[15] Karatzas, I., Shreve, S.: Brownian Motion and Stochastic Calculus, 2nd edn. Springer, Berlin (1991)

[16] Kato, T., Fujita, H.: On the nonstationary Navier-Stokes system. Rend. Semi. Math. Univ. Padova 32, 243-260 (1962)

[17] Kato, T.: Strong $L^{p}$-solutions of the Navier-Stokes equation in $R^{n}$, with applications to weak solutions. Math. Z. 187, 471-480 (1984)

[18] Kim, J.: On the stochastic wave equation with nonlinear damping. Appl. Math. Optim 58, 29-67 (2008)

[19] Mikulevicius, R., Rozovskii, B.L.: Global $L_{2}$-solutions of stochastic NavierStokes equations. Ann. Prob. 33, 137-176 (2005)

[20] Miyakawa, T.: On nonstationary solution of the Navier-Stokes equations in an exterior domain. Hiroshima Math. J. 12, 115-140 (1982)

[21] van Neerven, J., Veraar, M., Weis, L.: Maximal $L^{p}$ - regularity for stochastic evolution equations. SIAM Math. Anal 44, 1372-1414 (2012)

[22] Pazy, A.: Semigroups of Linear Operators and Applications to Partial Differential Equations. Springer, Berlin (1983)

[23] Seidler, J., Sobukawa, T.: Exponential integrability of stochastic convolutions. J. Lond. Math. Soc. 67, L245-258 (2003) 
[24] Sritharan, S., Sundar, P.: Large deviation for the two dimensional Navier-Stokes equations with multiplicative noise. Stoch. Pro. Appl. 116, 1636-1659 (2006)

[25] Sohr, H.: The Navier-Stokes Equations: An Elementary Functional Analysis Approach. Birkhauser, Boston (2001)

[26] Taniguchi, T.: The existence and decay estimates of the solutions to 3D stochastic Naver-Stokes equations with additive noise in an exterior domain, Discrete and Continuous Dynamical Systems-A (to appear)

[27] Taniguchi, T.: The existence of energy solutions to 2-dimensional nonLipschitz stochastic Navier-Stokes equations in unbounded domains. J. Diff. Equ. 251, 3329-3362 (2011)

[28] Temam, R.: Navier-Stokes Equation. North-Holland, Amsterdam (1977)

[29] Temam, R.: Infinite Dimensional Dynamical Systems in Mechanics and Physics, 2nd edn. Springer, New York

Takeshi Taniguchi

Division of Mathematical Sciences

Graduate School of Comparative Culture

Kurume University

Miimachi

Kurume

Fukuoka 839-8502

Japan

e-mail: takeshi_taniguchi@kurume-u.ac.jp

Received: 10 October 2013.

Accepted: 5 March 2014. 